\title{
The role of synaptic transmission in a HIV model with memory
}

\author{
Carla M.A. Pinto ${ }^{\star b}$, Ana R.M. Carvalho ${ }^{b}$, \\ ${ }^{a}$ School of Engineering, Polytechnic of Porto, \\ Rua Dr António Bernardino de Almeida, 431, 4200-072 Porto, Portugal \\ cap@isep.ipp.pt \\ ${ }^{b}$ Faculty of Sciences, University of Porto, \\ Rua do Campo Alegre s/n, 4440-452 Porto, Portugal \\ up200802541@fc.up.pt
}

\begin{abstract}
We propose a mathematical model with memory for the dynamics of HIV epidemics, where two transmission modes, cell-to-cell and virus-to-cell, and drug resistance are considered. Systems with memory, or fractional order systems, have largely been applied to the modelling of several real life phenomena. Here we consider a fractional model where the order of the noninteger derivative takes values in the interval $[0.5,1.0]$. We prove the local and global stability of the disease-free equilibrium. We study the role of the cell-to-cell transmission probability on the dynamics of the model, and on the value of the reproduction number, $R_{0}$, for distinct values of the fractional order derivative, $\alpha$. Moreover, we show evidence of an improvement of HIV infected patients quality of life, due to the increase of the drug efficacy. In the end, important inferences are drawn.

Keywords:

HIV, fractional order model, cell-to-cell transmission, treatment, drug-resistance
\end{abstract}

^ corresponding author 


\section{Introduction}

HIV infection constitutes a major challenge for public health worldwide, with more than 35 million HIV-1 infected people and more than two million new infections per year. In the EU/EEA, data from 2013, shows that there was an overall increase of $80 \%$ of new HIV diagnoses in comparison to 2004 [18]. Moreover, $47 \%$ of these cases were considered late diagnoses $\left(\mathrm{CD}^{+} \mathrm{T}\right.$ cell count $<350 / \mathrm{mm}^{3}$ ) and $27 \%$ were diagnosed with advanced HIV infection (CD4 $<200 / \mathrm{mm}^{3}$ ) [18]. In 2013, Portugal maintained the trend of decline in the number of new reported cases of HIV infection [34].

HIV acts in the human body by impairing the immune system and, in the absence of treatment, leads to death. HIV's main target are the leukocytes with $\mathrm{CD}^{+}{ }^{+}$receptors, known as $\mathrm{CD} 4^{+} \mathrm{T}$ cells. These cells may infect other cells which enhances viral spread through intercellular structures, called virological synapses. Understanding the role of cell-to-cell transmission in HIV infection is extremely useful since the primary cause of progression to AIDS in HIV infected patients is the depletion of these cells. In cell-to-cell transmission, HIV infectiousness is thought to be $10^{2}$ to $10^{3}$ times greater than in virus-to-cell transmission [17]. In [10], the polarized morphology of the helper cells, the Env-CD4 coordinated adhesion, and viral transfer from infected to uninfected $\mathrm{T}$ cells, indicate that the virological synapses allow HIV to elude the immune response and to spread cleverly. This agrees with the work of Sattentau [36]. There is, though, still controversy as to the real contribution of cell-to-cell transmission to viremia [31]. It is suggested that viral markers, in experimental studies, may not give precise measures of infectiousness and drug efficacy, since when quantified exactly indicate that anti-viral therapy (ART) blocks proviral DNA and virus replication as effectively in both transmission modes. This is in accordance with [39], where protease inhibitors, a crucial component of ART, are shown to be effective against both transmission pathways. See also [1]. The somewhat contradicting results concerning cell-to-cell transmission may be explained by donor and target cell types. The later crucially determines the extent at which imposed barriers in donors' cells are surmounted by cell-to-cell transmission [43]. Recent work by Galloway and co-workers [15, 19] shows that cell-to-cell is mandatory to set off pyroptotic death of (more than 95\% of) quiescent CD4 T cells in lymphoid tissues. Pyroptosis causes T cell depletion and chronic inflammation. These two pathogenic events boost one another, in fact, dying cells send inflammatory signals which in turn attract more $\mathrm{T}$ cells' death. The 
chronic inflammatory process may also contribute to the persistence of the latent HIV reservoir. The latent reservoir is believed to be responsible for the low levels of viremia in HIV-infected patients, after many years under successful suppressive ART [13]. In this sense, it could be worth investigating the effects of pyroptosis in these patients. Something worth noting is that virus-to-cell transmission cannot start pyroptosis. Infected $\mathrm{T}$ cells are thus main agents in boosting progression to AIDS in untreated HIV infected patients.

In the last decades, several mathematical models have been proposed to study the dynamics of HIV infection in the case of virus-to-cell transmission $[21,30,12,7]$. The cell-to-cell or synaptic transmission mechanism is first studied in the mid 90's [20, 38]. Later on, in 2009, Weng et al [41] propose a model for HIV infection where the two transmission modes are considered. They show that cell-to-cell transmission is crucial for HIV infection and it is much more important than virus-to-cell transmission. Cell-to-cell transmission is shown to adversely affect the immune system, leading to treatment failure in individuals with risk factors and potentially contributes to viral persistence in 2011 [37]. Two years later, Komarova et al [23] show evidence of equal contributions to viremia of virus-to-cell and cell-to-cell transmission modes. Moreover, they analyse the role of cell-to-cell transmission during drug therapy [24]. They argue that it is not straightforward that synaptic transmission contributes to viral persistence during treatment. Understanding the interplay between cell-to-cell transmission and anti-viral treatment must definetely take into account the still unknown values of the probabilities of individual virions to infect $\mathrm{T}$ cells in the two modes of transmission.

\subsection{Fractional calculus}

Fractional Calculus (FC) has suffered a major development in the last few years. Important applications of FC can be found in many areas of science, from electrochemistry, to physics, fluid mechanics, mechanical systems, other areas of engineering, and biology (mostly epidemiology) [29, 6, 28, 33, 4, 32, 3, $42,14,26,8]$. Derivatives of fractional order involve more information about the system in study than integer order derivatives, that are local operators. This 'memory property is very useful in the modeling of several phenomena. The state of the infection at a given moment $t$ depends on the states before $t$, namely $t-1, t-2, \cdots$. In this sense, $\mathrm{FC}$ may help to distinguish distinct routes (i.e., dynamics) in (HIV) infection in different patients. In 2009, Ding and Ye [14] analyse a fractional-order (FO) model for HIV dynamics. They 
obtain a condition on the number of virus for an infection to occur and prove the local stability of this endemic equilibrium. In [4], Arafa et al propose a FO model for the primary phase of HIV infection. The Generalized Euler Method is applied to approximate the solutions of the FO model. The authors vary the number of free virus for distinct initial conditions and discuss its implications in the solutions of the model. In 2014, the same authors compare the solutions of a FO model and its integer counterpart with data from ten HIV infected patients. They find that the FO model's predictions of the patients' viremia were more accurate. Liu and Lu [26] include logistic growth of healthy and infected $\mathrm{CD} 4^{+} \mathrm{T}$ cells , time delay and cure rate in a $\mathrm{FO}$ model for HIV. They give conditions for the asymptotic stability of the disease-free and endemic equilibria, for a delay $\tau \geq 0$. Carvalho and Pinto [8] propose a FO model for the co-infection of malaria and HIV, where personal protection and vaccination against malaria are considered. The reproduction number and the stability of the infection-free state are calculated. The simulations of the model for distinct values of the FO derivative and of relevant parameters of the model, namely the degree of sexual activity due to malaria, the HIV related mortality due to co-infection, the level of personal protection against malaria, amongst others, are biologically meaningful.

In this paper, we propose a FO model for HIV dinamics, that includes virus-to-cell and cell-to-cell transmission modes, and sensitive and drugresistance virus strains. The model is a modified and improved version of the one proposed in $[40,21,41]$. In Section 2 we describe the model. We compute the reproduction number and the local stability of the disease-free equilibrium in Section 3. In Section 4, the global asymptotic stability (GAS) of the disease-free equilibrium is obtained. The sensitivity analysis of the model is performed in Section 5. In Section 6, the model is simulated and the results are presented and discussed. Finally, in Section 7, we state the final conclusions of this work.

\section{Description of model}

The model consists of five compartments, including the populations of uninfected $\mathrm{CD}^{+}{ }^{+} \mathrm{T}$ cells, $T$, drug-sensitive infected $\mathrm{T}$ cells, $T_{s}$, drug-resistant infected $\mathrm{T}$ cells, $T_{r}$, drug-sensitive infectious virus, $V_{s}$, and drug-resistant infectious virus, $V_{r}$.

The dynamics of the uninfected $T$ cells is as follows. The growth rate of healthy $T$ cells is represented by the first two terms in the correspond- 
ing equation, where $\Lambda$ is the source of new cells and $d$ is their mortality rate. In the absence of infection, these cells proliferate exponentially at a rate $r$, until reaching the carrying capacity $T_{\max }$. These cells are infected by infectious virus $V_{s}$ and $V_{r}$ with a probability $k_{1}$. Cell-to-cell infection is modeled by the probability $k_{2}$. Treatment (parameter $u_{1}$ ) is considered only on the drug-sensitive cells. A value of $u_{1}=1$ indicates full efficacy of the reverse transcriptase inhibitors (RTIs), on the other hand, a zero value represents absence of treatment (zero efficacy). Drug-resistance in $T$ cells results from the failure of RTIs in blocking the reverse transcriptase enzyme, thus facilitating replication of HIV retrovirus. The virus' resistant strain is less fitted to infect $T$ cells, this information is incorporated in the equations by parameter $\psi$. Analogously, parameter $\psi_{1}$ models the reduced capacity of drug-resistant HIV infected $T$ cells, $T_{s}$, to infect other $T$ cells.

The dynamics of infected $T$ cells, $T_{s}$ and $T_{r}$, are given by the second and third equations of the model, respectively. Parameter $u_{3}$ represents drug efficacy in blocking cell-to-cell virus transmission. Cells $T_{s}$ become drugresistant with a probability $u . T_{s}$ and $T_{r}$ cells die at a rate $\delta_{2}$.

Finally, the last two equations model the virus strains, $V_{s}$ and $V_{r}$, dynamics. These strains are produced by the corresponding infected $\mathrm{CD} 4^{+}$populations, with bursting sizes of drug-sensitive strain, $N_{s}$, and of drug-resistant strain, $N_{r}$. The drug efficacy is inserted in the equations by parameter $u_{2}$. This parameter gives the efficacy of the protease inhibitors (PIs) in blocking the cleavage of viral polypeptide into functional enzymes. Parameter $k_{3}$ is the virus loss constant due to infection of $\mathrm{CD} 4^{+} \mathrm{T}$ cells. The clearance rate is $c$. The integer nonlinear system describing the dynamics of the model is:

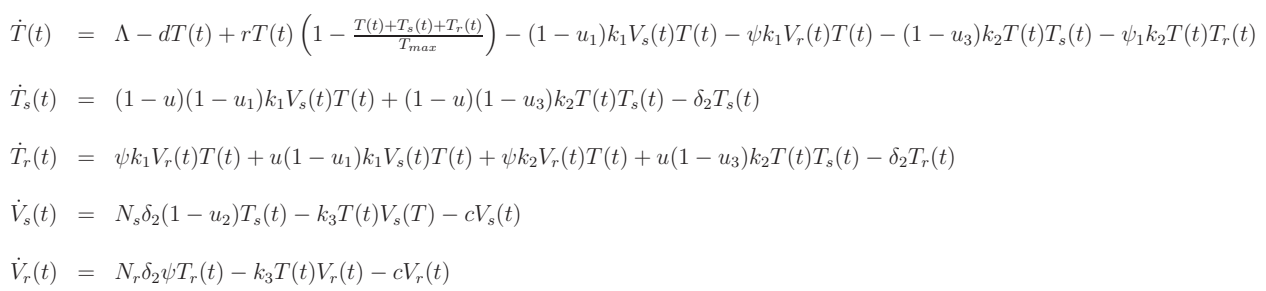

The fractional system corresponding to system (1) is as follows: 


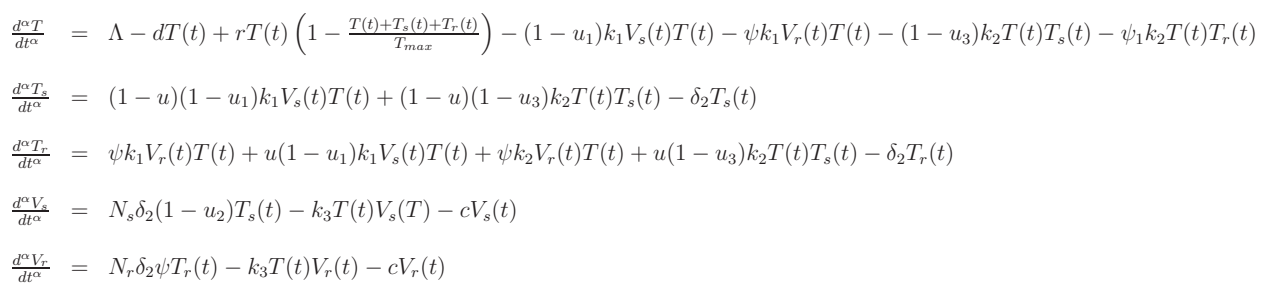

where $\alpha \in[0.5,1]$ is the order of the fractional derivative. When $\alpha=1$, the model is the integer order one. The fractional derivative of model (2) is used in the Caputo sense, i.e.:

$$
\frac{d^{\alpha} y(t)}{d t^{\alpha}}=I^{p-\alpha}-y^{(p)}(t), \quad t>0
$$

where $p=[\alpha]$ is the value of $\alpha$ rounded up to the nearest integer, $y^{(p)}$ is the $p$-th derivative of $y(r), I^{\star p}$ is the Riemman-Liouville operator of order $\star p>0$ given by:

$$
I^{p^{\star}} z(t)=\frac{1}{\Gamma\left(p^{\star}\right)} \int_{0}^{t}\left(t-t^{\prime}\right)^{p^{\star}-1} z\left(t^{\prime}\right) d t^{\prime}
$$

where $\Gamma\left(p^{\star}\right)$ is the gamma function.

A close observation of model (2) reveals some problems in time dimension. The left-hand side of the equations has dimension $(\text { time })^{-\alpha}$, whereas the right-hand side has dimension $(\text { time })^{-1}$. This handicap may be overcome and the corrected system corresponding to model (2) is written as [35]:

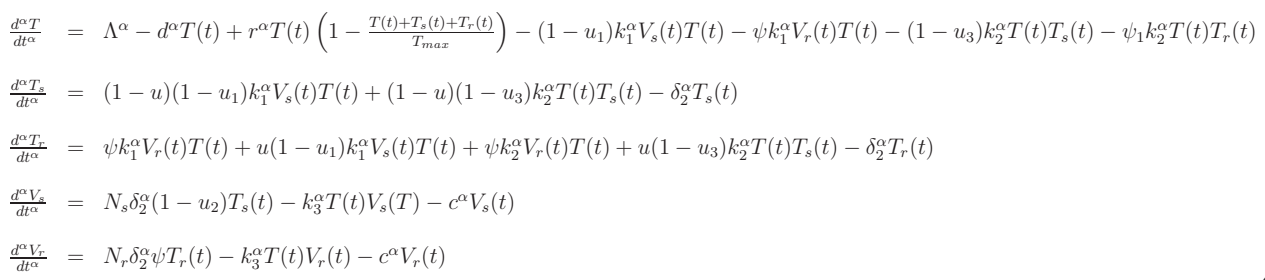

\section{Reproduction numbers and local stability of disease-free equi- librium}

In this subsection, we compute the reproduction number of model (3), $R_{0}$. The basic reproduction number is defined as the number of secondary infections due to a single infection in a completely susceptible population. 
We begin by considering two sub-models of model (1). Submodel (4) is derived from (3) by setting the variables concerning resistance dynamics $\left(T_{r}\right.$ and $V_{r}$ ) to zero, and model (8) follows from model (3) by setting the variables concerning sensitive dynamics $\left(T_{s}\right.$ and $\left.V_{s}\right)$ to zero.

We use the next generation method [16] to compute the reproduction number of system (4), $R_{s}$.

$$
\begin{aligned}
\frac{d^{\alpha} T}{d t^{\alpha}} & =\Lambda^{\alpha}+r^{\alpha} T(t)\left(1-\frac{T(t)+T_{s}(T)}{T_{\max }}\right)-d^{\alpha} T(t)-\left(1-u_{1}\right) k_{1}^{\alpha} V_{s}(t) T(t)-\left(1-u_{3}\right) k_{2}^{\alpha} T(t) T_{s}(t) \\
\frac{d^{\alpha} T_{s}}{d t^{\alpha}} & =(1-u)\left(1-u_{1}\right) k_{1}^{\alpha} V_{s}(t) T(t)+(1-u)\left(1-u_{3}\right) k_{2}^{\alpha} T(t) T_{s}(t)-\delta_{2}^{\alpha} T_{s}(t) \\
\frac{d^{\alpha} V_{s}}{d t^{\alpha}} & =N_{s} \delta_{2}^{\alpha}\left(1-u_{2}\right) T_{s}(t)-k_{3}^{\alpha} T(t) V_{s}(T)-c^{\alpha} V_{s}(t)
\end{aligned}
$$

The disease-free equilibrium of model (4) is given by:

$$
\begin{aligned}
P_{0}^{1} & =\left(T^{0}, T_{s}^{0}, V_{s}^{0}\right) \\
& =\left(\frac{T_{\max }\left[\left(r^{\alpha}-d^{\alpha}\right)+\sqrt{\left.\left(r^{\alpha}-d^{\alpha}\right)^{2}+\frac{4 r^{\alpha} \Lambda^{\alpha}}{T_{\max }}\right]}\right.}{2 r^{\alpha}}, 0,0\right)
\end{aligned}
$$

Using the notation in [16] on system (4), matrices for the new infection terms, $F_{s}$, and the other terms, $V_{s}$, are computed to be:

$$
\begin{gathered}
F_{s}=\left(\begin{array}{cc}
(1-u)\left(1-u_{3}\right) k_{2}^{\alpha} T^{0} & (1-u)\left(1-u_{1}\right) k_{1}^{\alpha} T^{0} \\
0 & 0
\end{array}\right) \\
V_{s}=\left(\begin{array}{cc}
\delta_{2}^{\alpha} & 0 \\
-N_{s} \delta_{2}^{\alpha}\left(1-u_{2}\right) & k_{3}^{\alpha} T^{0}+c^{\alpha}
\end{array}\right)
\end{gathered}
$$

The associative basic reproduction number is thus:

$$
R_{s}=\rho\left(F_{s} V_{s}^{-1}\right)=\frac{(1-u)\left(1-u_{3}\right) k_{2}^{\alpha} T^{0}}{\delta_{2}^{\alpha}}+\frac{N_{s}\left(1-u_{2}\right)(1-u)\left(1-u_{1}\right) k_{1}^{\alpha} T^{0}}{k_{3}^{\alpha} T^{0}+c^{\alpha}}
$$

where $\rho$ indicates the spectral radius of $F V^{-1}$.

Lemma 1. [27] The disease-free equilibrium $P_{0}^{1}$ is locally asymptotically stable if all eigenvalues $\lambda_{i}$ of the linearization matrix of model (4), satisfy $\left|\arg \left(\lambda_{i}\right)\right|>\alpha \frac{\pi}{2}$. 
Proof. The linearization matrix of model (4) around the disease-free equilibrium, $P_{0}^{1}$, is:

$$
M_{1}=\left(\begin{array}{ccc}
-\sqrt{\left(r^{\alpha}-d^{\alpha}\right)^{2}+\frac{4 r^{\alpha} \Lambda^{\alpha}}{T_{\max }}} & -\frac{r^{\alpha}}{T_{\max }} T^{0}-\left(1-u_{3}\right) k_{2}^{\alpha} T^{0} & -\left(1-u_{1}\right) k_{1}^{\alpha} T^{0} \\
0 & (1-u)\left(1-u_{3}\right) k_{2}^{\alpha} T^{0}-\delta_{2}^{\alpha} & (1-u)\left(1-u_{1}\right) k_{1}^{\alpha} T^{0} \\
0 & N_{s} \delta_{2}^{\alpha}\left(1-u_{2}\right) & -k_{3}^{\alpha} T^{0}-c^{\alpha}
\end{array}\right)
$$

The following eigenvalue is easily obtained $-\sqrt{\left(r^{\alpha}-d^{\alpha}\right)^{2}+\frac{4 r^{\alpha} \Lambda^{\alpha}}{T_{\max }}}$.

The remaining eigenvalues are the roots of the characteristic equation of a $2 \times 2$ matrix, $M_{2}$, given by:

$$
M_{2}=\left(\begin{array}{cc}
(1-u)\left(1-u_{3}\right) k_{2}^{\alpha} T^{0}-\delta_{2}^{\alpha} & (1-u)\left(1-u_{1}\right) k_{1}^{\alpha} T^{0} \\
N_{s}\left(1-u_{2}\right) \delta_{2}^{\alpha} & -k_{3}^{\alpha} T^{0}-c^{\alpha}
\end{array}\right)
$$

The determinant $\left|M_{2}-\lambda I_{2}\right|=0$ is equivalent to:

$$
\begin{aligned}
& \left((1-u)\left(1-u_{3}\right) k_{2}^{\alpha} T^{0}-\delta_{2}^{\alpha}-\lambda\right)\left(-k_{3}^{\alpha} T^{0}-c^{\alpha}-\lambda\right)-N_{s}\left(1-u_{2}\right) \delta_{2}^{\alpha}(1-u)\left(1-u_{1}\right) k_{1}^{\alpha} T^{0}=0 \\
& \lambda^{2}+\left(-(1-u)\left(1-u_{3}\right) k_{2}^{\alpha} T^{0}+\delta_{2}^{\alpha}+k_{3}^{\alpha} T^{0}+c^{\alpha}\right) \lambda+\left((1-u)\left(1-u_{3}\right) k_{2}^{\alpha} T^{0}-\delta_{2}^{\alpha}\right)\left(-k_{3}^{\alpha} T^{0}-c^{\alpha}\right)- \\
& -N_{s}\left(1-u_{2}\right) \delta_{2}^{\alpha}(1-u)\left(1-u_{1}\right) k_{1}^{\alpha} T^{0}=0
\end{aligned}
$$

Thus, the characteristic polynomial is:

$$
P_{1}(\lambda)=\lambda^{2}+a_{1} \lambda+a_{2}=0
$$

where

$$
\begin{aligned}
& a_{1}=-(1-u)\left(1-u_{3}\right) k_{2}^{\alpha} T^{0}+\delta_{2}^{\alpha}+k_{3}^{\alpha} T^{0}+c^{\alpha} \\
& a_{2}=-\left((1-u)\left(1-u_{3}\right) k_{2}^{\alpha} T^{0}-\delta_{2}^{\alpha}\right)\left(k_{3}^{\alpha} T^{0}+c^{\alpha}\right)-N_{s}\left(1-u_{2}\right) \delta_{2}^{\alpha}(1-u)\left(1-u_{1}\right) k_{1}^{\alpha} T^{0}
\end{aligned}
$$

The disease-free equilibrium $P_{0}^{1}$ is locally asymptotically stable for $\alpha \in[0,1)$ if $\left|\arg \left(\lambda_{i}\right)\right|>\alpha \frac{\pi}{2}, \forall i=1,2,3$. Following Ahmed et al [2], the Routh-Hurwitz conditions are necessary and sufficient for $\left|\arg \left(\lambda_{i}\right)\right|>\alpha \frac{\pi}{2}$. In this case, we must verify that $a_{1}, a_{2}>0$.

It is easy to show that:

$$
a_{1}=-(1-u)\left(1-u_{3}\right) k_{2}^{\alpha} T^{0}+\delta_{2}^{\alpha}+k_{3}^{\alpha} T^{0}+c^{\alpha}=T^{0}\left(k_{3}^{\alpha}-(1-u)\left(1-u_{3}\right) k_{2}^{\alpha}\right)+\left(\delta_{2}^{\alpha}+c^{\alpha}\right)>0
$$


We now proceed with the proof of $a_{2}>0$.

$$
\begin{aligned}
& -\left((1-u)\left(1-u_{3}\right) k_{2}^{\alpha} T^{0}-\delta_{2}^{\alpha}\right)\left(k_{3}^{\alpha} T^{0}+c^{\alpha}\right)-N_{s}\left(1-u_{2}\right) \delta_{2}^{\alpha}(1-u)\left(1-u_{1}\right) k_{1}^{\alpha} T^{0}>0 \\
& \Leftrightarrow-\left((1-u)\left(1-u_{3}\right) k_{2}^{\alpha} T^{0}-\delta_{2}^{\alpha}\right)-\frac{N_{s}\left(1-u_{2}\right) \delta_{2}^{\alpha}(1-u)\left(1-u_{1}\right) k_{1}^{\alpha} T^{0}}{k_{3}^{\alpha} T^{0}+c^{\alpha}}>0 \\
& \Leftrightarrow-\frac{(1-u)\left(1-u_{3}\right) k_{2}^{\alpha} T^{0}}{\delta_{2}^{\alpha}}+1-\frac{N_{s}\left(1-u_{2}\right)(1-u)\left(1-u_{1}\right) k_{1}^{\alpha} T^{0}}{k_{3}^{\alpha} T^{0}+c^{\alpha}}>0 \Leftrightarrow 1-R_{s}>0 \Leftrightarrow R_{s}<1
\end{aligned}
$$

Thus, the disease-free equilibrium $P_{0}^{1}$ is locally asymptotically stable for $\alpha \in$ $[0,1)$.

We proceed with the computation of the reproduction number of model (8), $R_{r}$, below.

$$
\begin{aligned}
\frac{d^{\alpha} T}{d t^{\alpha}} & =\Lambda^{\alpha}+r^{\alpha} T(t)\left(1-\frac{T(t)+T_{r}(T)}{T_{\max }}\right)-d^{\alpha} T(t)-\psi k_{1}^{\alpha} V_{r}(t) T(t)-\psi_{1} k_{2}^{\alpha} T(t) T_{r}(t) \\
\frac{d^{\alpha} T_{r}}{d t^{\alpha}} & =\psi k_{1}^{\alpha} V_{r}(t) T(t)+\psi_{1} k_{2}^{\alpha} T(t) T_{r}(t)-\delta_{2}^{\alpha} T_{r}(t) \\
\frac{d^{\alpha} V_{r}}{d t^{\alpha}} & =N_{r} \delta_{2}^{\alpha} \psi T_{r}(t)-k_{3}^{\alpha} T(t) V_{r}(t)-c^{\alpha} V_{r}(t)
\end{aligned}
$$

The disease-free equilibrium state, $P_{0}^{2}$, of model (8) is given by:

$$
\begin{aligned}
P_{0}^{2} & =\left(T^{0}, T_{r}^{0}, V_{r}^{0}\right) \\
& =\left(T^{0}, 0,0\right)
\end{aligned}
$$

Using the notation in [16] on system (8), matrices for the new infection terms, $F_{r}$, and the other terms, $V_{r}$, are given by:

$$
\begin{gathered}
F_{r}=\left(\begin{array}{cc}
\psi_{1} k_{2}^{\alpha} T^{0} & \psi k_{1}^{\alpha} T^{0} \\
0 & 0
\end{array}\right) \\
V_{r}=\left(\begin{array}{cc}
\delta_{2}^{\alpha} & 0 \\
-N_{r} \delta_{2}^{\alpha} \psi & k_{3}^{\alpha} T^{0}+c^{\alpha}
\end{array}\right)
\end{gathered}
$$

The associative basic reproduction number is computed to be:

$$
R_{r}=\rho\left(F_{r} V_{r}^{-1}\right)=\frac{\psi_{1} k_{2}^{\alpha} T^{0}}{\delta_{2}^{\alpha}}+\frac{N_{r} \psi^{2} k_{1}^{\alpha} T^{0}}{k_{3}^{\alpha} T^{0}+c^{\alpha}}
$$

where $\rho$ indicates the spectral radius of $F V^{-1}$. 
Lemma 2. [27] The disease-free equilibrium $P_{0}^{2}$ is locally asymptotically stable if all eigenvalues $\lambda_{i}$ of the linearization matrix of model (8), satisfy $\left|\arg \left(\lambda_{i}\right)\right|>\alpha \frac{\pi}{2}$.

Proof. The matrix of the linearization of model (8) around the diseasefree equilibrium, $P_{0}^{2}$, is calculated to be:

$$
M_{3}=\left(\begin{array}{ccc}
-\sqrt{\left(r^{\alpha}-d^{\alpha}\right)^{2}+\frac{4 r^{\alpha} \Lambda^{\alpha}}{T_{\max }}} & -\frac{r^{\alpha}}{T_{\max }} T^{0}-\psi_{1} k_{2}^{\alpha} T^{0} & -\psi k_{1}^{\alpha} T^{0} \\
0 & \psi_{1} k_{2}^{\alpha} T^{0}-\delta_{2}^{\alpha} & \psi k_{1}^{\alpha} T^{0} \\
0 & N_{r} \delta_{2}^{\alpha} \psi & -k_{3}^{\alpha} T^{0}-c^{\alpha}
\end{array}\right)
$$

The following eigenvalue is easily obtained $-\sqrt{\left(r^{\alpha}-d^{\alpha}\right)^{2}+\frac{4 r^{\alpha} \Lambda^{\alpha}}{T_{\max }}}$.

The remaining eigenvalues are the roots of the characteristic equation of matrix $M_{4}$, given by:

$$
M_{4}=\left(\begin{array}{cc}
\psi_{1} k_{2}^{\alpha} T^{0}-\delta_{2}^{\alpha} & \psi k_{1}^{\alpha} T^{0} \\
N_{r} \delta_{2}^{\alpha} \psi & -k_{3}^{\alpha} T^{0}-c^{\alpha}
\end{array}\right)
$$

The determinant $\left|M_{4}-\lambda I_{2}\right|=0$ is equivalent to:

$$
\begin{aligned}
& \left(\psi_{1} k_{2}^{\alpha} T^{0}-\delta_{2}^{\alpha}-\lambda\right)\left(-k_{3}^{\alpha} T^{0}-c^{\alpha}-\lambda\right)-N_{r} \delta_{2}^{\alpha} \psi^{2} k_{1}^{\alpha} T^{0}=0 \\
& \lambda^{2}+\left(-\psi_{1} k_{2}^{\alpha} T^{0}+\delta_{2}^{\alpha}+k_{3}^{\alpha} T^{0}+c^{\alpha}\right) \lambda-\left(\psi_{1} k_{2}^{\alpha} T^{0}-\delta_{2}^{\alpha}\right)\left(k_{3}^{\alpha} T^{0}+c^{\alpha}\right)-N_{r} \delta_{2}^{\alpha} \psi^{2} k_{1}^{\alpha} T^{0}=0
\end{aligned}
$$

The associated characteristic polynomial is:

$$
P_{1}(\lambda)=\lambda^{2}+a_{1} \lambda+a_{2}=0
$$

where

$$
\begin{aligned}
& a_{1}=-\psi_{1} k_{2}^{\alpha} T^{0}+\delta_{2}^{\alpha}+k_{3}^{\alpha} T^{0}+c^{\alpha} \\
& a_{2}=-\left(\psi_{1} k_{2}^{\alpha} T^{0}-\delta_{2}^{\alpha}\right)\left(k_{3}^{\alpha} T^{0}+c^{\alpha}\right)-N_{r} \delta_{2}^{\alpha} \psi^{2} k_{1}^{\alpha} T^{0}
\end{aligned}
$$

The disease-free equilibrium $P_{0}^{2}$ is locally asymptotically stable for $\alpha \in[0,1)$ if $\left|\arg \left(\lambda_{i}\right)\right|>\alpha \frac{\pi}{2}, \forall i=1,2,3$. Ahmed et al [2] show that the usual RouthHurwitz conditions $a_{1}, a_{2}>0$, are necessary and sufficient for $\left|\arg \left(\lambda_{i}\right)\right|>\alpha \frac{\pi}{2}$. We prove that $a_{1}>0$. 


$$
a_{1}=-\psi_{1} k_{2}^{\alpha} T^{0}+\delta_{2}^{\alpha}+k_{3}^{\alpha} T^{0}+c^{\alpha}=T^{0}\left(k_{3}^{\alpha}-\psi_{1} k_{2}^{\alpha}\right)+\left(\delta_{2}^{\alpha}+c^{\alpha}\right)>0
$$

We proceed with the proof of $a_{2}>0$.

$$
\begin{aligned}
& -\left(\psi_{1} k_{2}^{\alpha} T^{0}-\delta_{2}^{\alpha}\right)\left(k_{3}^{\alpha} T^{0}+c^{\alpha}\right)-N_{r} \delta_{2}^{\alpha} \psi^{2} k_{1}^{\alpha} T^{0}>0 \Leftrightarrow-\left(\psi_{1} k_{2}^{\alpha} T^{0}-\delta_{2}^{\alpha}\right)-\frac{N_{r} \alpha_{\alpha}^{\alpha} \psi k_{1}^{\alpha} T^{0}}{k_{3}^{2} T^{0}+c^{\alpha}}>0 \\
& \Leftrightarrow-\frac{\psi_{1} k_{2}^{\alpha} T^{0}}{\delta_{2}^{\alpha}}+1-\frac{N_{r} \psi^{2} k_{1}^{\alpha} T^{0}}{k_{3}^{\alpha} T^{0}+c^{\alpha}}>0 \Leftrightarrow 1-R_{r}>0 \Leftrightarrow R_{r}<1
\end{aligned}
$$

Thus, the disease-free equilibrium $P_{0}^{2}$ is locally asymptotically stable for $\alpha \in$ $[0,1)$.

The reproduction number of the full model (3), $R_{0}$ is calculated as follows. The disease-free equilibrium state, $P_{0}$, of model (1) is given by:

$$
\begin{aligned}
P_{0} & =\left(T^{0}, T_{s}^{0}, T_{r}^{0}, V_{s}^{0}, V_{r}^{0}\right) \\
& =\left(T^{0}, 0,0,0,0\right)
\end{aligned}
$$

Using the notation in [16] on system (1), matrices for the new infection terms, $F$, and the other terms, $V$, are given by:

$$
\begin{gathered}
F=\left(\begin{array}{cccc}
(1-u)\left(1-u_{3}\right) k_{2}^{\alpha} T^{0} & 0 & (1-u)\left(1-u_{1}\right) k_{1}^{\alpha} T^{0} & 0 \\
u\left(1-u_{3}\right) k_{2}^{\alpha} T^{0} & \psi_{1} k_{2}^{\alpha} T^{0} & u\left(1-u_{1}\right) k_{1}^{\alpha} T^{0} & \psi k_{1}^{\alpha} T^{0} \\
0 & 0 & 0 & 0 \\
0 & 0 & 0 & 0
\end{array}\right) \\
V=\left(\begin{array}{cccc}
\delta_{2}^{\alpha} & 0 & 0 & 0 \\
0 & \delta_{2}^{\alpha} & 0 & 0 \\
-N_{s} \delta_{2}^{\alpha}\left(1-u_{2}\right) & 0 & k_{3}^{\alpha} T^{0}+c^{\alpha} & 0 \\
0 & -N_{r} \delta_{2}^{\alpha} \psi & 0 & k_{3}^{\alpha} T^{0}+c^{\alpha}
\end{array}\right)
\end{gathered}
$$

The associative basic reproduction number is computed to be:

$$
R_{0}=\rho\left(F V^{-1}\right)=\max \left\{R_{s}, R_{r}\right\}
$$

where $\rho$ indicates the spectral radius of $F V^{-1}$. 
Lemma 3. [27] The disease-free equilibrium $P_{0}$ is locally asymptotically stable if all eigenvalues $\lambda_{i}$ of the linearization matrix of model (3), satisfy $\left|\arg \left(\lambda_{i}\right)\right|>\alpha \frac{\pi}{2}$.

Proof. The linearization matrix of model (3) around the disease-free equilibrium $P_{0}$ is given by:

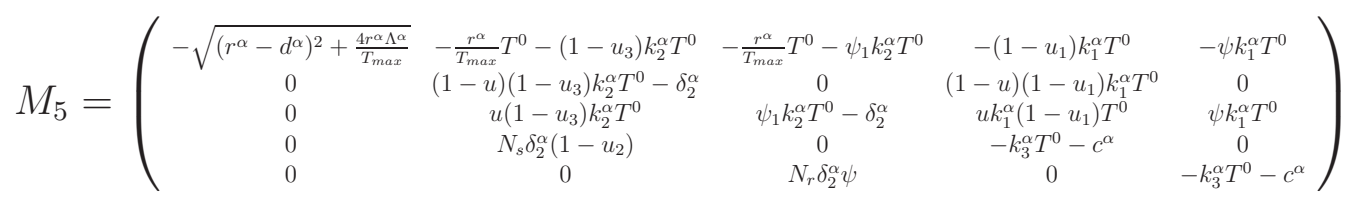

The following eigenvalue is easily obtained $-\sqrt{\left(r^{\alpha}-d^{\alpha}\right)^{2}+\frac{4 r^{\alpha} \Lambda^{\alpha}}{T_{\max }}}$.

The remaining eigenvalues are the roots of the characteristic equation of matrix $M_{6}$, below.

$$
M_{6}=\left(\begin{array}{cccc}
(1-u)\left(1-u_{3}\right) k_{2}^{\alpha} T^{0}-\delta_{2}^{\alpha} & 0 & (1-u)\left(1-u_{1}\right) k_{1}^{\alpha} T^{0} & 0 \\
u\left(1-u_{3}\right) k_{2}^{\alpha} T^{0} & \psi_{1} k_{2}^{\alpha} T^{0}-\delta_{2}^{\alpha} & u k_{1}^{\alpha}\left(1-u_{1}\right) T^{0} & \psi k_{1}^{\alpha} T^{0} \\
N_{s} \delta_{2}^{\alpha}\left(1-u_{2}\right) & 0 & -k_{3}^{\alpha} T^{0}-c^{\alpha} & 0 \\
0 & N_{r} \delta_{2}^{\alpha} \psi & 0 & -k_{3}^{\alpha} T^{0}-c^{\alpha}
\end{array}\right)
$$

The determinant $\left|M_{6}-\lambda I_{4}\right|=0$ is equivalent to:

$$
\begin{aligned}
& \left((1-u)\left(1-u_{3}\right) k_{2}^{\alpha} T^{0}-\delta_{2}^{\alpha}-\lambda\right)\left|\begin{array}{ccc}
\psi_{1} k_{2}^{\alpha} T^{0}-\delta_{2}^{\alpha}-\lambda & u k_{1}^{\alpha}\left(1-u_{1}\right) T^{0} & \psi k_{1}^{\alpha} T^{0} \\
0 & -k_{3}^{\alpha} T^{0}-c^{\alpha}-\lambda & 0 \\
N_{r} \delta_{2}^{\alpha} \psi & 0 & -k_{3}^{\alpha} T^{0}-c^{\alpha}-\lambda
\end{array}\right|+
\end{aligned}
$$

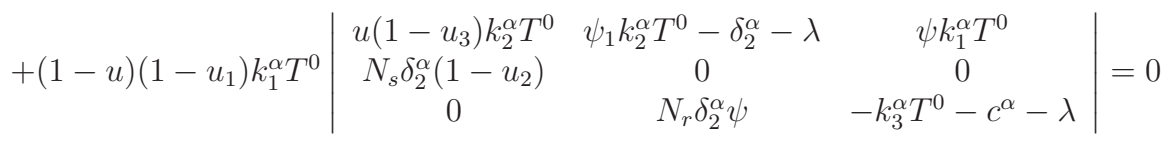

$$
\begin{aligned}
& \left((1-u)\left(1-u_{3}\right) k_{2}^{\alpha} T^{0}-\delta_{2}^{\alpha}-\lambda\right)\left(-k_{3}^{\alpha} T^{0}-c^{\alpha}-\lambda\right)\left|\begin{array}{cc}
\psi_{1} k_{2}^{\alpha} T^{0}-\delta_{2}^{\alpha}-\lambda & \psi k_{1}^{\alpha} T^{0} \\
N_{r} \delta_{2}^{\alpha} \psi & -k_{3}^{\alpha} T^{0}-c^{\alpha}-\lambda
\end{array}\right|- \\
& -N_{s} \delta_{2}^{\alpha}\left(1-u_{2}\right)(1-u)\left(1-u_{1}\right) k_{1}^{\alpha} T^{0}\left|\begin{array}{cc}
\psi_{1} k_{2}^{\alpha} T^{0}-\delta_{2}^{\alpha}-\lambda & \psi k_{1}^{\alpha} T^{0} \\
N_{r} \delta_{2}^{\alpha} \psi & -k_{3}^{\alpha} T^{0}-c^{\alpha}-\lambda
\end{array}\right|=0 \\
& {\left[\left((1-u)\left(1-u_{3}\right) k_{2}^{\alpha} T^{0}-\delta_{2}^{\alpha}-\lambda\right)\left(-k_{3}^{\alpha} T^{0}-c^{\alpha}-\lambda\right)-N_{s} \delta_{2}^{\alpha}\left(1-u_{2}\right)(1-u)\left(1-u_{1}\right) k_{1}^{\alpha} T^{0}\right] \times} \\
& \times\left[\left(\psi_{1} k_{2}^{\alpha} T^{0}-\delta_{2}^{\alpha}-\lambda\right)\left(-k_{3}^{\alpha} T^{0}-c^{\alpha}-\lambda\right)-N_{r} \delta_{2}^{\alpha} \psi^{2} k_{1}^{\alpha} T^{0}\right]=0
\end{aligned}
$$


The first factor of the characteristic equation of matrix $M_{6}$ is the characteristic equation of matrix $M_{2}$, and the second factor is the characteristic equation of the matrix $M_{4}$. Thus, as we have already proven that the disease free equilibria of subsystems (4)-(8) are stable for $R_{s}<1$ and $R_{r}<1$, respectively, one concludes that the disease free equilibrium $P_{0}$ is locally asymptotically stable for $R_{0}=\max \left\{R_{s}, R_{r}\right\}<1$.

\section{Global stability of disease-free equilibria}

In this subsection, we compute the GAS of the disease free equilibrium of the full model (3). Again, we begin by the stability of the disease free equilibria of the two submodels.

Lemma 4. The disease free equilibrium $P_{0}^{1}$ is $G A S$ if $R_{s}<1$.

\section{Proof.}

Consider the following Lyapunov function.

$$
L_{1}=T_{s}+\frac{(1-u)\left(1-u_{1}\right) k_{1}^{\alpha} T^{0}}{k_{3}^{\alpha} T^{0}+c^{\alpha}} V_{s}
$$

The derivative of $L_{1}$ along a solution of system (4) is:

$$
\begin{aligned}
\dot{L_{1}}= & \dot{T}_{s}+\frac{(1-u)\left(1-u_{1}\right) k_{1}^{\alpha} T^{0}}{k_{3}^{\alpha} T^{0}+c^{\alpha}} \dot{V}_{s} \\
= & (1-u)\left(1-u_{1}\right) k_{1}^{\alpha} V_{s} T+(1-u)\left(1-u_{3}\right) k_{2}^{\alpha} T T_{s}-\delta_{2}^{\alpha} T_{s}+ \\
& +\frac{N_{s} \delta_{2}^{\alpha}\left(1-u_{2}\right)(1-u)\left(1-u_{1}\right) k_{1}^{\alpha} T^{0}}{k_{3}^{\alpha} T^{0}+c^{\alpha}} T_{s}-(1-u)\left(1-u_{1}\right) k_{1}^{\alpha} T^{0} V_{s} \frac{k_{3}^{\alpha} T+c_{3}^{\alpha} T^{0}+c^{\alpha}}{k^{\alpha}} \\
= & (1-u)\left(1-u_{1}\right) k_{1}^{\alpha}\left(T-T^{0} \frac{k_{3}^{\alpha} T+c^{\alpha}}{k_{3}^{\alpha} T^{0}+c^{\alpha}}\right) V_{s}+\left((1-u)\left(1-u_{3}\right) k_{2}^{\alpha} T-\delta_{2}^{\alpha}+\right. \\
& \left.+\delta_{2}^{\alpha} \frac{N_{s}\left(1-u_{2}\right)(1-u)\left(1-u_{1}\right) k_{1}^{\alpha} T^{0}}{k_{3}^{\alpha} T^{0}+c^{\alpha}}\right) T_{s} \\
= & (1-u)\left(1-u_{1}\right) k_{1}^{\alpha} \frac{k_{3}^{\alpha} T T^{0}+c^{\alpha} T-k_{3}^{\alpha} T T^{0}-c^{\alpha} T^{0}}{k_{3}^{\alpha} T^{0}+c^{\alpha}} V_{s}+ \\
& +\left((1-u)\left(1-u_{3}\right) k_{2}^{\alpha} T-\delta_{2}^{\alpha}+\delta_{2}^{\alpha}\left(R_{s}-\frac{(1-u)\left(1-u_{3}\right) k_{2}^{\alpha} T^{0}}{\delta_{2}^{\alpha}}\right)\right) T_{s} \\
= & (1-u)\left(1-u_{1}\right) k_{1}^{\alpha} \frac{c^{\alpha}\left(T-T^{0}\right)}{k_{3}^{\alpha} T^{0}+c^{\alpha}} V_{s}+(1-u)\left(1-u_{3}\right) k_{2}^{\alpha}\left(T-T^{0}\right) T_{s}+\delta_{2}^{\alpha}\left(R_{s}-1\right) T_{s} \\
\leq & \delta_{2}^{\alpha}\left(R_{s}-1\right) T_{s}
\end{aligned}
$$


since $T(t) \leq T^{0}$. Thus, when $R_{s}<1$, we have $\dot{L}_{1} \leq 0$. Furthermore, $\dot{L}_{1}=0$ if and only if $T=T^{0}, T_{s}=V_{s}=0$, which means that the maximum invariant set in $\left\{\left(T, T_{s}, V_{s}\right) \in \Gamma_{s}: \dot{L_{1}}=0\right\}$ is $\left\{P_{0}^{1}\right\}$. Using LaSalles Invariance Principle [25] we conclude that all solutions in $\Gamma_{s}$ converge to $P_{0}^{1}$. The global attractiveness of $P_{0}^{1}$ and its local stability imply its global stability.

We now repeat the same procedure for the proof of the GAS of the diseasefree equilibrium of submodel (8).

Lemma 5. The disease-free equilibrium $P_{0}^{2}$ is GAS if $R_{r}<1$.

Proof. We set the Lyapunov function below.

$$
L_{2}=T_{r}+\frac{\psi k_{1}^{\alpha} T^{0}}{k_{3}^{\alpha} T^{0}+c^{\alpha}} V_{r}
$$

The derivative of $L_{2}$ along the solution of system (8) is:

$$
\begin{aligned}
\dot{L_{2}} & =\dot{T}_{r}+\frac{\psi k_{1}^{\alpha} T^{0}}{k_{3}^{\alpha} T^{0}+c^{\alpha}} \dot{V}_{r} \\
& =\psi k_{1}^{\alpha} T V_{r}+\psi_{1} k_{2}^{\alpha} T T_{r}-\delta_{2}^{\alpha} T_{r}+\frac{N_{r} \delta_{2}^{\alpha} \psi^{2} k_{1}^{\alpha} T^{0}}{k_{3}^{\alpha} T^{0}+c^{\alpha}} T_{r}-\psi k_{1}^{\alpha} T^{0} V_{r} \frac{k_{3}^{\alpha} T+c^{\alpha}}{k_{3}^{\alpha} T^{0}+c^{\alpha}} \\
& =\psi k_{1}^{\alpha}\left(T-T^{0} \frac{k_{3}^{\alpha} T+c^{\alpha}}{k_{3}^{\alpha} T^{0}+c^{\alpha}}\right) V_{r}+\left(\psi_{1} k_{2}^{\alpha} T-\delta_{2}^{\alpha}+\delta_{2}^{\alpha} \frac{N_{r} \psi^{2} k_{1}^{\alpha} T^{0}}{k_{3}^{\alpha} T^{0}+c^{\alpha}}\right) T_{r} \\
& =\psi k_{1}^{\alpha} V_{r} \frac{k_{3}^{\alpha} T^{0} T+c^{\alpha} T-k_{3}^{\alpha} T^{0} T-c^{\alpha} T^{0}}{k_{3}^{\alpha} T^{0}+c^{\alpha}}+\left(\psi_{1} k_{2}^{\alpha} T-\delta_{2}^{\alpha}+\delta_{2}^{\alpha}\left(R_{r}-\frac{\psi_{1} k_{2}^{\alpha} T^{0}}{\delta_{2}^{\alpha}}\right)\right) T_{r} \\
& =\psi k_{1}^{\alpha} V_{r} \frac{c^{\alpha}\left(T-T^{0}\right)}{k_{3}^{\alpha} T^{0}+c^{\alpha}}+\psi_{1} k_{2}^{\alpha}\left(T-T^{0}\right) T_{r}+\delta_{2}^{\alpha}\left(R_{r}-1\right) T_{r} \\
& \leq \delta_{2}^{\alpha}\left(R_{r}-1\right) T_{r}
\end{aligned}
$$

since $T(t) \leq T^{0}$. When $R_{r}<1$, we obtain $\dot{L_{2}} \leq 0$. Moreover, $\dot{L_{2}}=0$ if and only if $T=T^{0}, T_{r}=V_{r}=0$, which means, that the maximum invariant set in $\left\{\left(T, T_{r}, V_{r}\right) \in \Gamma_{r}: \dot{L_{2}}=0\right\}$ is $\left\{P_{0}^{2}\right\}$. Using LaSalles Invariance Principle [25] we conclude that all solutions in $\Gamma_{r}$ converge to $P_{0}^{2}$. The global attractiveness of $P_{0}^{2}$ and its local stability imply its global stability.

We end this section with the proof of the GAS of the disease-free equilibrium of the full model (3). 
Lemma 6. The disease-free equilibrium $P_{0}$ is $G A S$ if $\max \left\{R_{s}, R_{r}\right\}<1$.

Proof. The Lyapunov function is assumed to be:

$$
L=L_{1}+L_{2}
$$

The derivative of $L$ along the solution of system (3) is:

$$
\begin{aligned}
\dot{L}= & \dot{L_{1}}+\dot{L_{2}} \\
= & (1-u)\left(1-u_{1}\right) k_{1}^{\alpha} V_{s} T+(1-u)\left(1-u_{3}\right) k_{2}^{\alpha} T T_{s}-\delta_{2}^{\alpha} T_{s}+ \\
& +\frac{N_{s} \delta_{2}^{\alpha}\left(1-u_{2}\right)(1-u)\left(1-u_{1}\right) k_{1}^{\alpha} T^{0}}{k_{3}^{\alpha} T^{0}+c^{\alpha}} T_{s}-(1-u)\left(1-u_{1}\right) k_{1}^{\alpha} T^{0} V_{s} \frac{k_{s}^{\alpha} T+c^{\alpha} T^{0}+c^{\alpha}}{k^{2}}+ \\
& \psi k_{1}^{\alpha} T V_{r}+\psi_{1} k_{2}^{\alpha} T T_{r}-\delta_{2}^{\alpha} T_{r}+\frac{N_{r} \delta_{2}^{\alpha} \psi^{2} k_{1}^{\alpha} T^{0}}{k_{3}^{\alpha} T^{0}+c^{\alpha}} T_{r}-\psi k_{1}^{\alpha} T^{0} V_{r} \frac{k_{3}^{\alpha} T+c^{\alpha}}{k_{3}^{\alpha} T^{0}+c^{\alpha}} \\
= & (1-u)\left(1-u_{1}\right) k_{1} \frac{c^{\alpha}\left(T-T^{0}\right)}{k_{3}^{\alpha} T^{0}+c^{\alpha}} V_{s}+(1-u)\left(1-u_{3}\right) k_{2}^{\alpha}\left(T-T^{0}\right) T_{s}+\delta_{2}^{\alpha}\left(R_{s}-1\right) T_{s}+ \\
& +\psi k_{1}^{\alpha} V_{r} \frac{c^{\alpha}\left(T-T^{0}\right)}{k_{3}^{\alpha} T^{0}+c^{\alpha}}+\psi_{1} k_{2}^{\alpha}\left(T-T^{0}\right) T_{r}+\delta_{2}^{\alpha}\left(R_{r}-1\right) T_{r} \\
\leq & \delta_{2}^{\alpha}\left(\left(R_{s}-1\right) T_{s}+\left(R_{r}-1\right) T_{r}\right)
\end{aligned}
$$

since $T(t) \leq T^{0}$. Thus, when $R_{s}, R_{r}<1$, we obtain $\dot{L} \leq 0$. Furthermore, $\dot{L}=0$ if and only if $T=T^{0}, T_{s}=T_{r}=V_{s}=V_{r}=0$, which means, that the maximum invariant set in $\left\{\left(T, T_{s}, T_{r}, V_{s}, V_{r}\right) \in \Gamma: \dot{L}=0\right\}$ is $\left\{P_{0}\right\}$. Using LaSalles Invariance Principle [25] we can conclude that all solutions in $\Gamma$ converge to $P_{0}$. The global attractiveness of $P_{0}$ and its local stability imply its global stability. The proof is completed.

\section{Sensitivity Analysis}

In this section we perform the sensitivity analysis of the reproduction number, $R_{0}$, to relevant parameters of model (3). This analysis measures the relative change in a variable subjected to the variation of a given parameter. We follow the procedure developed in [11]. Measuring the $R_{0}$ sensitivity indexes is extremely important since the value of $R_{0}$ determines the spread or the eradication of the disease.

The calculations are done for the parameter values given in Table 2. The signs of the sensitivity indices may be found in Table 7. A positive sign indicates an increase in the value of $R_{0}$ as the parameter is increased. Inversely 
a negative sign of the index means that $R_{0}$ decreases as the parameter is increased. For example, for $\alpha=1$, an increase of $10 \%$ in the value of $k_{2}$ increases $R_{0}$ by $3.4 \%$. Similarly for the other indexes.

\section{Table 1 more or less here}

\section{Numerical Results}

We simulate model (3). The parameters used in the simulations are given in Table 2. The initial conditions are set to $T(0)=10^{6} \mathrm{~mL}^{-1}, T_{s}(0)=$ $10^{3} \mathrm{~mL}^{-1}$, and all other variables to $10 \mathrm{~mL}^{-1}$. The order of the fractional derivative was varied in the interval $[0.5,1.0]$ with steps of 0.1 .

Table 2 more or less here

In Figures 1-6, we depict the dynamics of model (3) for different values of the cell-to-cell transmission probability $k_{2}$, and of the fractional order derivative ( $\alpha=1, \alpha=0.7$ and $\alpha=0.5$ ). We notice that as $k_{2}$ is increased it is observed an aggravation of the health status of HIV infected patients, for all values of $\alpha$. In particular, in Figures 1-2, it is depicted an endemic state for the two values of $k_{2}$. The values of $R_{0}$ are $R_{0}=24$, for $k_{2}=3 \times 10^{-5}$, and $R_{0}=2.40$ for $k_{2}=3 \times 10^{-6}$. Thus, as $k_{2}$ decreases, the endemic state severity is reduced, with greater values of the total and uninfected CD $4^{+}$ $\mathrm{T}$ cells, for smaller values of $k_{2}$. In Figures 3-4, are depicted an endemic state for $k_{2}=0.1 \times 10^{-6}$, and a disease-free equilibrium for $k_{2}=0.2 \times 10^{-8}$. The values of $R_{0}$ are $R_{0}=5.55$, for $k_{2}=0.1 \times 10^{-6}$, and $R_{0}=0.37$ for $k_{2}=0.2 \times 10^{-8}$. In this case, it is shown numerically a bifurcation from an endemic state to a disease-free state, as $k_{2}$ is decreased. In the graphs of Figures 5-6, are observed two endemic states. The values of $R_{0}$ are $R_{0}=9.43$, for $k_{2}=7 \times 10^{-10}$, and $R_{0}=1.53$ for $k_{2}=1 \times 10^{-11}$. Once again, for larger values of $k_{2}$, the endemic state is more severe, with larger numbers of sensitive virus and infected $T$ cells. Another interesting feature that comes out of these figures is that the value of the fractional order derivative, $\alpha$, may also contribute to the severity of the disease. The values of $R_{0}$ increase, despite the decrease on $k_{2}$, as $\alpha$ decreases from 1 . This may help to distinguish distinct progression routes of HIV infected patients, namely associated with patients' immune system status, genetic profile, and age [5], for example.

Figure 1 more or less here

Figure 2 more or less here

Figure 3 more or less here

Figure 4 more or less here 
Figure 5 more or less here

Figure 6 more or less here

In Figures 7-9, it is shown the variation of the reproduction number $R_{0}$, of model (3), as a function of the probabilities $k_{1}$ and $k_{2}$, and $\alpha . R_{0}$ increases monotonically both with $k_{1}$ and $k_{2}$. Moreover, a closer look also reveals that even when $k_{1}=0$, if $k_{2}$ is large enough, then $R_{0}$ may be greater than 1 . This pattern is observed for all values of the fractional order derivative, $\alpha$ and agrees with the results of Figs 1-6, as expected. Since a value of $R_{0}$ greater than 1 indicates a disease state, and a value of $R_{0}$ smaller than 1 is associated with a disease-free condition, then $k_{2}$ has a major effect on the progression of the infection.

Figure 7 more or less here

Figure 8 more or less here

Figure 9 more or less here

In Figures 10-11, we depict the dynamics of system (3) for distinct values of the treatment efficacy, $u_{1}$, and for $\alpha=1.0$. We observe that as the drugefficacy $u_{1}$ is increased, there is an improvement in HIV infected patients quality of life, due to a decrease in infectiousness, associated with smaller values of infected cells and virus. For $u_{1}=0.2$, the model exhibits an endemic state, whereas for $u_{1}=0.6$ we observe a disease-free equilibrium. The corresponding reproduction numbers are $R_{0}=1.01$ and $R_{0}=0.99$. This behaviour, concerning the effect of drug-efficacy in the epidemics, is seen for other values of $\alpha$. In the sake of completeness, we note that for smaller values of $\alpha$ the variables approach faster the corresponding asymptotic values. This is a common feature of the FO systems, since they use more information from the past behaviour of the system to simulate future states, than integer-order models. This feature could help devise more adaptable HIV patients therapies in what concerns dosage or intervals of administration. Are well known the sometimes severe secondary effects of ART, and better therapies could improve the quality of life of treated HIV-1 infected patients.

Figure 10 more or less here

Figure 11 more or less here

Overall, cell-to-cell transmission has an important role in the development of HIV infection and strategies should be developed in order to block it. 


\section{Conclusion}

We propose a FO model for the dynamics of HIV epidemics with two transmission modes and drug-resistance. The order of the fractional derivative, $\alpha$, takes values in the interval $[0.5,1]$. We prove that for $R_{0}<1$, the disease-free equilibrium is GAS. When $R_{0}>1$, the disease-free equilibrium becomes unstable and the endemic equilibrium gains stability. We show that the cell-to-cell transmission rate has a considerable impact in the value of $R_{0}$, for all values of $\alpha$. This is consistent with biological findings of HIV epidemics in vivo, where at the level of the lymph nodes, cell-to-cell transmission is crucial for the development of the disease [41]. The cell-to-cell HIV pathway sets off a stronger immune response than that of the virus-to-cell in lymphoid tissues' latenty infected $\mathrm{CD}^{+} \mathrm{T}$ cells $[15,19]$. These latently infected cells die by pyroptosis, a strong inflammatory form of cell death, which is due to the accumulation of incomplete cytosolic viral DNA intermediates in their inside. This inflammatory mechanism contributes to the latent HIV reservoir, which in turn is responsible for the persistence of low levels of viremia in successfully treated HIV infected patients [13]. Pyroptosis is only possible in cell-to-cell transmission mode. The later highlights the extremely important role of the cell-cell pathway in the progression to AIDS in HIV infected patients. The order of the fractional derivative may be used, in this sense, to explain differences in the progression routes of HIV infected patients, associated with patients' immune system status, specific features in donor and target cell types, genetic profile, age [5, 43].

We also simulate the effect of treatment in the dynamics of the model. We observe that an increase of the drug efficacy, in a given parameter region, translates in a better quality of life for HIV infected patients, since the infectiousness is reduced. This agrees with the results from $[39,1]$, in which effective ART regimens are shown to suppress viremia at cell-cell contact sites. The increase in the treatment efficacy produces similar results for all values of the FO derivative, $\alpha$. Nevertheless, there are differences in the form (faster for smaller $\alpha$ ) in which the variables approach the asymptotic values. This could be used to adjust HIV patients' therapies in what concerns dosage or intervals of administration, which in turn might reduce the well known side effects of the drugs.

All to all, there is still much to be understood in terms of the mechanisms behind cell-to-cell transmission, persistence and establishment of viral reservoirs in order to devise better therapies and aim to cure AIDS [9]. A 
very recent study suggests that maybe this cure is closer than expected. Gene editing using clustered, regularly-interspaced, short palindromic repeats (CRISPR)/CRISPR-associated 9 (Cas9) nuclease is proposed as a new therapeutic approach for HIV eradication from $\mathrm{CD} 4^{+} \mathrm{T}$ cells and as a possible cure for AIDS [22]. This type of therapeutic approach targets the donor and not the virus itself and is has been considered promising [15].

Future work will verify the conclusions of the proposed model for data of Portuguese HIV infected patients.

\section{Acknowledgments}

Authors wish to thank the Polytechnic of Porto, through the PAPRE Programa de Apoio à Publicação em Revistas Científicas de Elevada Qualidade for financial support. The authors were partially funded by the European Regional Development Fund through the program COMPETE and by the Portuguese Government through the FCT - Fundação para a Ciência e a Tecnologia under the project PEst-C/MAT/UI0144/2013. The research of AC was partially supported by a FCT grant with reference SFRH/BD/96816/2013.

\section{References}

[1] L.M. Agosto, P. Zhong, J. Munro, W. Mothes, Highly Active Antiretroviral Therapies Are Effective against HIV-1 Cell-to-Cell Transmission, PLoS Pathog 10(2) (2014) e1003982.

[2] E. Ahmed, A.M.A. El-Sayed, H.A.A. El-Saka, On some Routh-Hurwitz conditions for fractional order differential equations and their applications in Lorenz, Rössler, Chua and Chen systems, Phys. Lett. A 358 (2006) 1-4.

[3] A.A.M. Arafa, S. Z. Rida, and M. Khalil, A fractional-order model of HIV infection: Numerical solution and comparisons with data of patients, Int. J. Biomath. 07 (2014) 1450036.

[4] A.A.M. Arafa, S.Z. Rida, M. Khalil, Fractional modeling dynamics of HIV and CD4 ${ }^{+}$T-cells during primary infection, Nonlinear Biomed. Phys. 6(1) (2012) 1. 
[5] S.H. Bajaria, G. Webb, M. Cloyd, D. Kirschner, Dynamics of naive and memory $\mathrm{CD}^{+} \mathrm{T}$ lymphocytes in HIV-1 disease progression, J. AIDS 30 (2002) 41-58.

[6] M. Caputo, F. Mainardi, A new dissipation model based on memory mechanism, Pure Appl. Geophys. 91 (8) (1971) 134-147.

[7] A.R.M. Carvalho, C.M.A. Pinto, A coinfection model for HIV and HCV, BioSystems 124 (2014) 46-60.

[8] A.R.M. Carvalho, C.M.A. Pinto, A delay fractional order model for the co-infection of malaria and HIV/AIDS, Int. J. Dynam. Control, First online: 05 February 2016, DOI 10.1007/s40435-016-0224-3.

[9] N. Casartelli, HIV-1 Cell-to-Cell Transmission and Antiviral Strategies: An Overview, Curr. Drug Targets. 17(1) (2016) 65-75.

[10] P. Chen, W. Hübner, M.A. Spinelli, B.K. Chen, Predominant Mode of Human Immunodeficiency Virus Transfer between T Cells Is Mediated by Sustained Env-Dependent Neutralization-Resistant Virological Synapses, J Virol. 81(22) (2007) 12582-12595.

[11] N. Chitnis, J.M. Hyman, J.M. Cushing, Determining important parameters in the spread of malaria through the sensitivity analysis of a mathematical model, B. Math. Biol. 70 (2008) 1272-1296.

[12] J.M. Conway, A.S. Perelson, Post-treatment control of HIV infection, Proc. Natl. Acad. Sci. U.S.A. 112(17) (2015) 5467-5472.

[13] J.M. Conway, A.S. Perelson, Residual Viremia in Treated HIV+ Individuals, PLoS Comput. Biol. 12(1) (2016) e1004677.

[14] Y. Ding, H. Ye, A fractional-order differential equation model of HIV infection of CD4 ${ }^{+}$T-cells, Math. Comput. Model. 50(3-4) (2009) 386392.

[15] G. Doitsh, N.L. Galloway, X. Geng, Z. Yang, K.M. Monroe, O. Zepeda, P.W. Hunt, H. Hatano, S. Sowinski, I. Munoz-Arias, W.C. Greene, Pyroptosis drives CD4 T-cell depletion in HIV-1 infection, Nature 505(7484) (2014) 509-514. 
[16] P. Driessche, P. Watmough, Reproduction numbers and sub-threshold endemic equilibria for compartmental models of disease transmission, Math. Biosci. 180 (2002) 29-48.

[17] D.S. Dimitrov, R.L. Willey, H. Sato, L.-J. Chang, R. Blumenthal, M.A. Martin, Quantitation of human immunodeficiency virus type 1 infection kinetics, J. Virol. 67 (1993) 2182-2190.

[18] European Centre for Disease Prevention and Control, HIV/AIDS surveillance in Europe, Surveillance Report, http://ecdc.europa.eu/en/publications/Publications/ hiv-aids-surveillance-report-Europe-2013.pdf (accessed 21.06.16).

[19] N.L. Galloway, G. Doitsh, K.M. Monroe, Z. Yang, I. Munoz-Arias, D.N. Levy, W.C. Greene, Cell-to-cell transmission of HIV-1 is required to trigger pyroptotic death of lymphoid tissue-derived CD4 T cells. Cell Rep. 12(10) (2015) 1555-1563.

[20] P. Gupta, R. Balachandran, Cell-to-cell transmission of humman immunodefficiency virus type 1 in the presence of azidothymidine and neutralizing antibody, J. Virol. 63 (1989) 2361-2365.

[21] M.M. Hadjiandreou, R. Conejeros, D.I. Wilson, Long-term HIV dynamics subject to continuous therapy and structured treatment interruptions, Chem. Eng. Sci. 64 (2009) 1600-1617.

[22] R. Kaminski, Y. Chen, T. Fischer, E. Tedaldi, A. Napoli, Y. Zhang, J. Karn, W. Hu, K. Khalil, Elimination of HIV-1 Genomes from Human T-lymphoid Cells by CRISPR/Cas9 Gene Editing, Sci. Rep. 6 (2016) 22555.

[23] N.L. Komarova, D. Anghelina, I. Voznesensky, B. Trinité, D.N. Levy, D. Wodarz, Relative contribution of free-virus and synaptic transmission to the spread of HIV-1 through target cell populations, Biol. Lett. 9(1) (2013) 20121049.

[24] N.L. Komarova, D. Levy, D. Wodarz, Synaptic transmission and the susceptibility of HIV infection to anti-viral drugs, Sci. Rep. 3 (2013) 2103. 
[25] J.P. LaSalle, The Stability of Dynamical Systems, SIAM, Philadelphia, 1976.

[26] Z. Liu, P. Lu, Stability analysis for HIV infection of CD4+ T-cells by a fractional differential time-delay model with cure rate, Adv. Difference Equ. 2014 (2014) 298.

[27] D. Matignon, Stability results for fractional differential equations with applications to control processing. in: Computational Engineering in Systems Applications 2, Lille, France, 1996, pp. 963-968.

[28] S. Momani, Z. Odibat, Analytical approach to linear fractional partial differential equations arising in fluid mechanics, Phys. Lett. A 355(45) (2006) 271-279.

[29] K.B. Oldham, Fractional differential equations in electrochemistry, Adv. Eng. Softw. 41(1) (2010) 9-12.

[30] A.S. Perelson, R.M. Ribeiro, Modeling the within-host dynamics of HIV infection, BMC Biology 11(1) (2013) 96.

[31] M. Permanyer, E. Ballana, A. Ruiz, R. Badia, E. Riveira-Munoz, E. Gonzalo, B. Clotet, J.A. Esté, Antiretroviral Agents Effectively Block HIV Replication after Cell-to-Cell Transfer, J. Virol. 86(16) (2012) 87738780 .

[32] C.M.A. Pinto, A.R.M. Carvalho, New findings on the dynamics of HIV and TB coinfection models, Appl. Math. Comput. 242 (2014) 36-46.

[33] C.M.A. Pinto, J.A. Tenreiro Machado, Fractional central pattern generators for bipedal locomotion, Nonlinear Dynam. 620(12) (2010) 27-37.

[34] Programa Nacional para a Infeção VIH/SIDA, Portugal - Infeção VIH, SIDA e Tuberculose em números 2014. file:///C:/Users/cpinto/ Downloads/i021017.pdf (accessed 21.06.16).

[35] T. Sardar, S. Rana, S. Bhattacharya, K. Al-Khaled, J. Chattopadhyay, A generic model for a single strain mosquito-transmitted disease memory on the host and the vector, Math. Biosci. 263 (2015) 18-36.

[36] Q. Sattentau, Avoiding the void: cell-to-cell spread of human viruses, Nat. Rev. Microbiol. 6(11) (2008) 815-826. 
[37] A. Sigal, J.T. Kim, A.B. Balazs, E. Dekel, A. Mayo, R. Milo, D. Baltimore, Cell-to-cell spread of HIV permits ongoing replication despite antiretroviral therapy, Nature 477(7362) (2011) 95-98.

[38] J.L. Spouge, R.I. Shrager, D.S. Dimitrov, HIV-1 infection kinetics in tissue cultures, Math. Biosci. 138(1) (1996) 1-22.

[39] B.K. Titanji, M. Aasa-Chapman, D. Pillay, C. Jolly, Protease inhibitors effectively block cell-to-cell spread of HIV-1 between T cells, Retrovirology 10 (1) (2013) 161.

[40] Y. Wang, F. Brauer, J. Wu, J.M. Heffernan, A delay-dependent model with HIV drug resistance during therapy, J. Math. Anal. Appl. 414 (2014) 514-531.

[41] Q. Wen, J. Lou, The global dynamics of a model about HIV-1 infection in vivo, Ric. Mat. 58 (2009) 77-90.

[42] H. Ye, Y. Ding, Nonlinear Dynamics and Chaos in a Fractional-Order HIV Model, Math. Probl. Eng. 2009 (2009) Article ID 378614, 12 pages.

[43] P. Zhong, L.M. Agosto, A. Ilinskaya, B. Dorjbal, R. Truong, D. Derse, P.D. Uchil, G. Heidecker, W. Mothes, Cell-to-Cell Transmission Can Overcome Multiple Donor and Target Cell Barriers Imposed on CellFree HIV, PLoS ONE 8(1) (2013) e53138. 
Table 1: Parameters used in the numerical simulations of model (3).

\begin{tabular}{|c|c|c|c|}
\hline \multicolumn{2}{|c|}{ Parameter } & \multicolumn{2}{|c|}{ Sensitivity index sign } \\
\hline$k_{1}$ & + & & \\
\hline$k_{2}$ & + & & \\
\hline$k_{3}$ & - & & \\
\hline$u$ & - & & \\
\hline$u_{1}^{\alpha}$ & - & & \\
\hline$u_{2}^{\alpha}$ & - & & \\
\hline$u_{3}^{\alpha}$ & - & & \\
\hline$\delta_{2}^{\alpha}$ & - & & \\
\hline$c^{\alpha}$ & - & & \\
\hline$N_{s}^{\alpha}$ & + & & \\
\hline$N_{r}^{\alpha}$ & + & & \\
\hline$\psi^{\alpha}$ & + & & \\
\hline$\psi_{1}^{\alpha}$ & + & & \\
\hline Parameter & Value & Units & Reference \\
\hline$\lambda$ & $10^{4}$ & cells $\mathrm{mL}^{-1}$ day $^{-1}$ & [12] \\
\hline$d$ & 0.01 & day $^{-1}$ & {$[12]$} \\
\hline$\delta_{2}$ & 1 & day $^{-1}$ & {$[12]$} \\
\hline$r$ & 0.03 & day $^{-1}$ & {$[12]$} \\
\hline$c$ & 23 & day $^{-1}$ & {$[12]$} \\
\hline$T_{\max }$ & $10^{6}$ & $\mathrm{~mL}$ & {$[12]$} \\
\hline$u_{1}$ & 0.2 & & {$[40]$} \\
\hline$u_{2}$ & 0.2 & & {$[40]$} \\
\hline$u_{3}$ & 0.7 & & Estimated \\
\hline$u$ & $3 \times 10^{-5}$ & & {$[21]$} \\
\hline$\psi$ & 0.5 & & {$[21]$} \\
\hline$\psi_{1}$ & 0.1 & & {$[21]$} \\
\hline$k_{1}$ & $0.1 \times 10^{-11}$ & $\mathrm{~mL}^{-1} \mathrm{day}^{-1}$ & {$[12]$} \\
\hline$k_{2}$ & $2.4 \times 10^{-7}$ & $m L^{-1}$ day $^{-1}$ & {$[41]$} \\
\hline$k_{3}$ & $8.79 \times 10^{-9}$ & $\mathrm{~mL}^{-1} \mathrm{day}^{-1}$ & {$[21]$} \\
\hline$N_{s}$ & 200 & & {$[40]$} \\
\hline$N_{r}$ & 50 & & {$[40]$} \\
\hline
\end{tabular}

Table 2: Parameters used in the numerical simulations of model (3). 

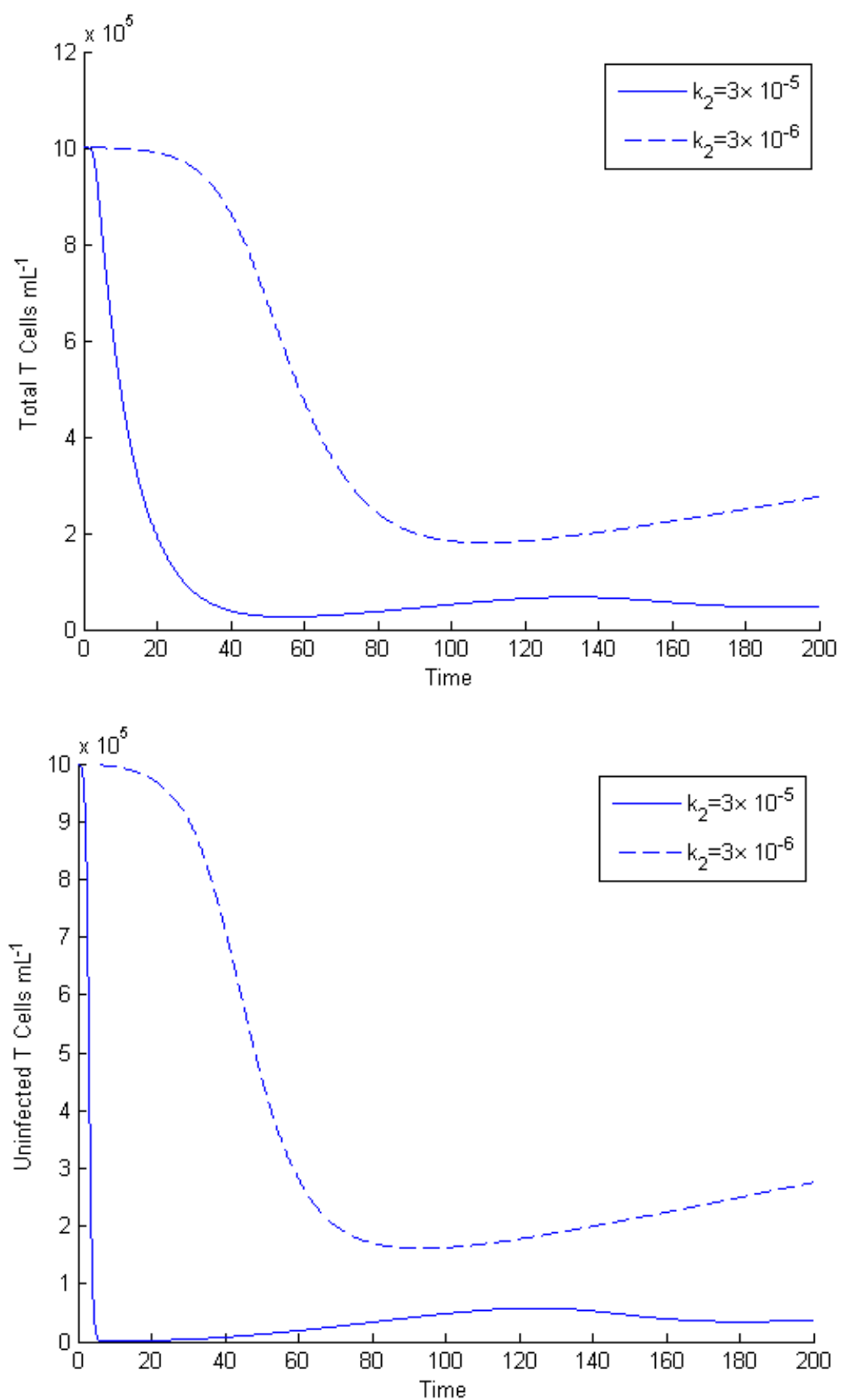

Figure 1: Dynamics of the the total number and uninfected $T$ cells of system (3) for different values of $k_{2}$, the probability of cell-to-cell transmission, for $\alpha=1$, given parameter values in Table 2, and given initial conditions. For more information, see text. 

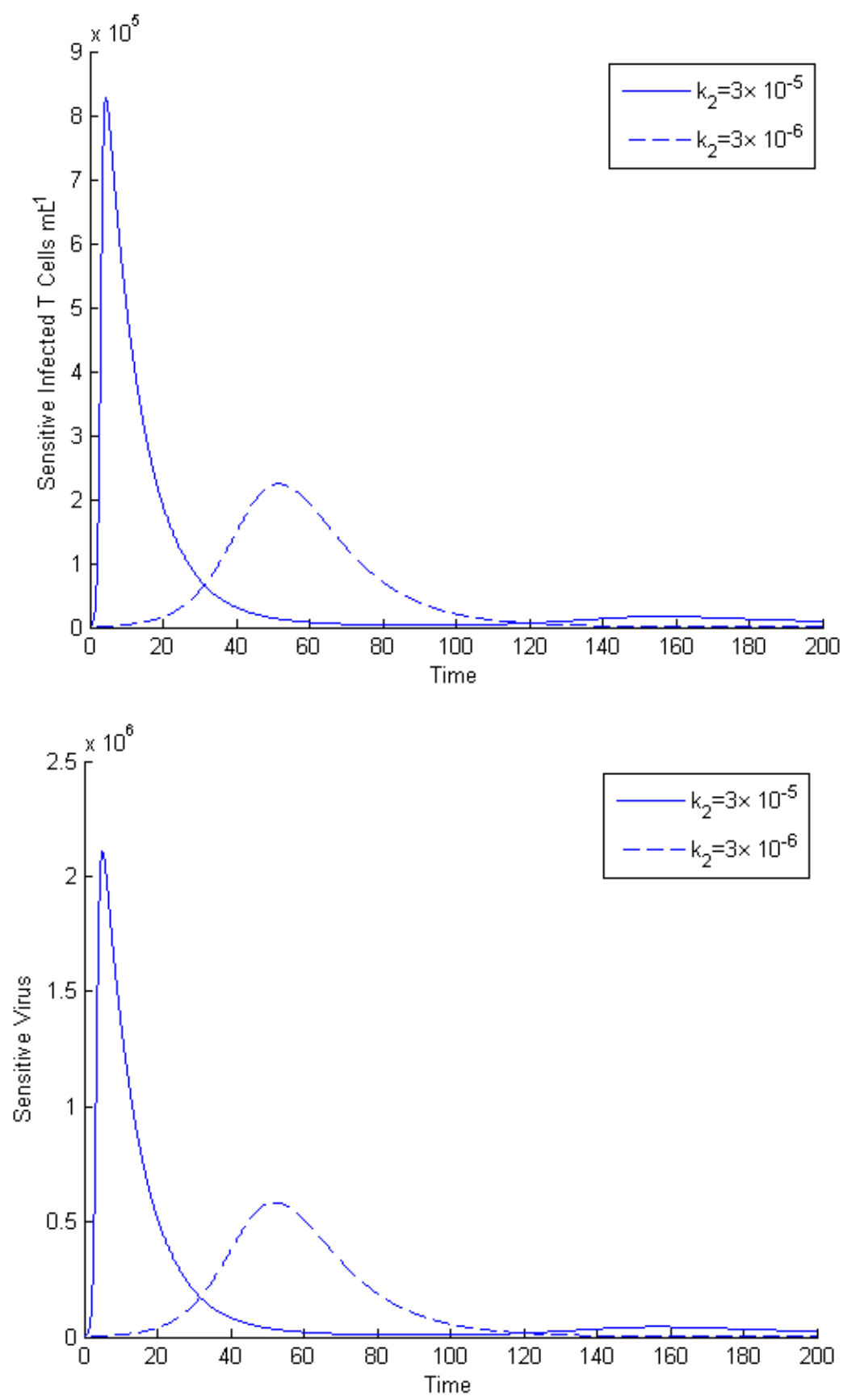

Figure 2: Dynamics of the sensitive $T$ cells and virus of system (3) for different values of $k_{2}$, the probability of cell-to-cell transmission, for $\alpha=1$, given parameter values in Table 2 , and given initial conditions. For more information, see text. 

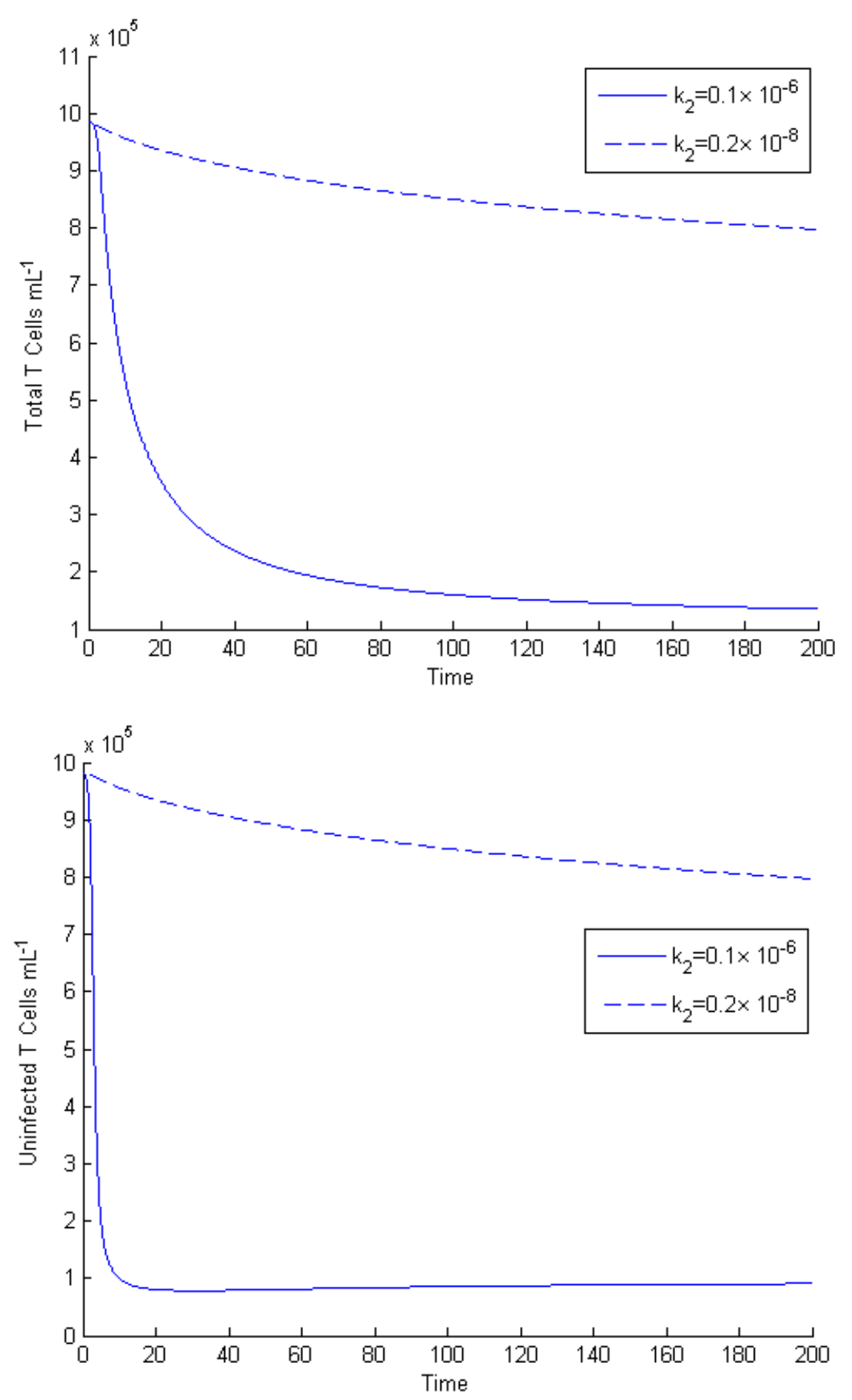

Figure 3: Dynamics of the total number and uninfected $T$ cells of system (3) for different values of $k_{2}$, the probability of cell-to-cell transmission, for $\alpha=0.7$, given parameter values in Table 2, and given initial conditions. For more information, see text. 

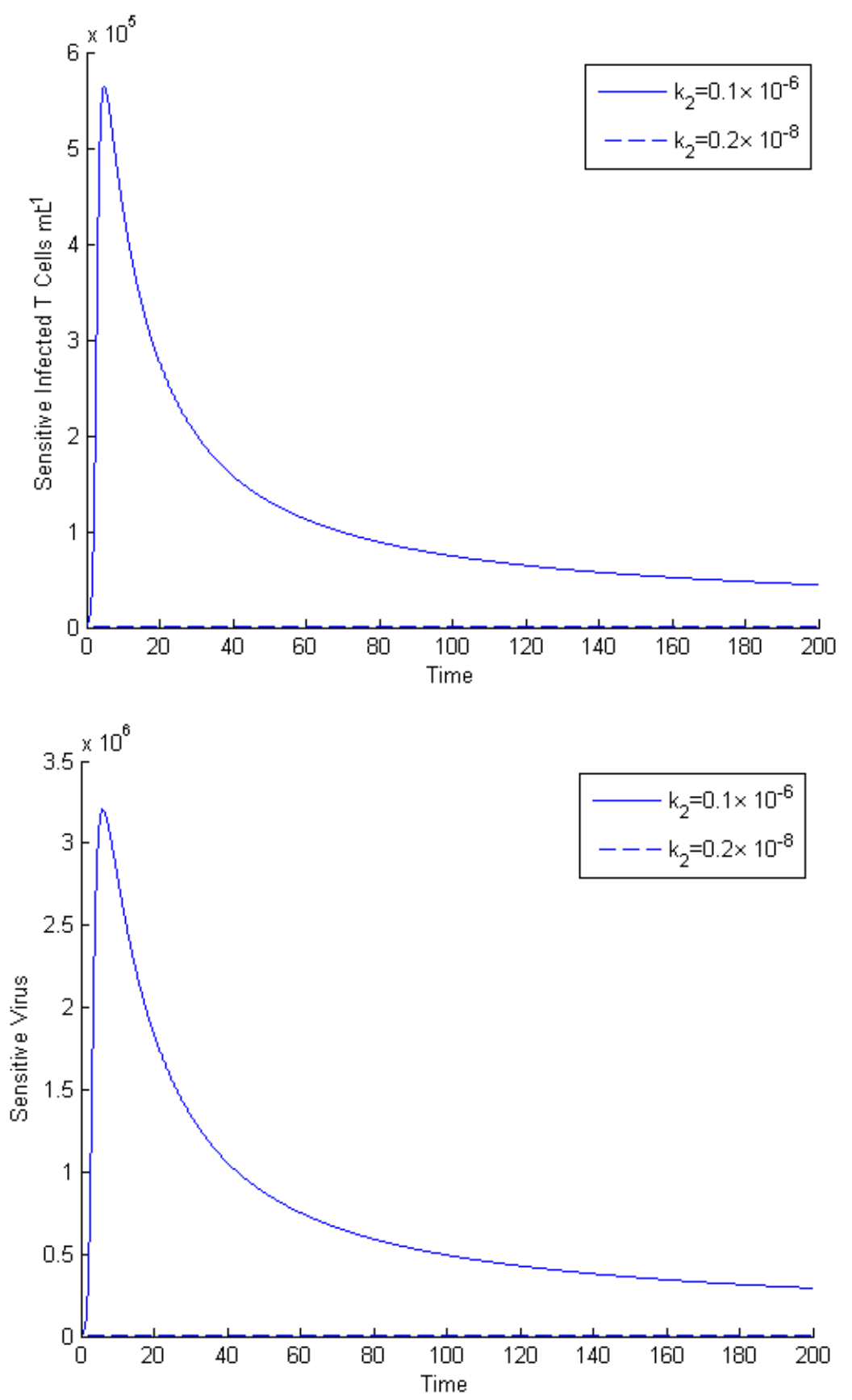

Figure 4: Dynamics of the sensitive $T$ cells and virus of system (3) for different values of $k_{2}$, the probability of cell-to-cell transmission, for $\alpha=0.7$, given parameter values in Table 2, and given initial conditions. For more information, see text. 

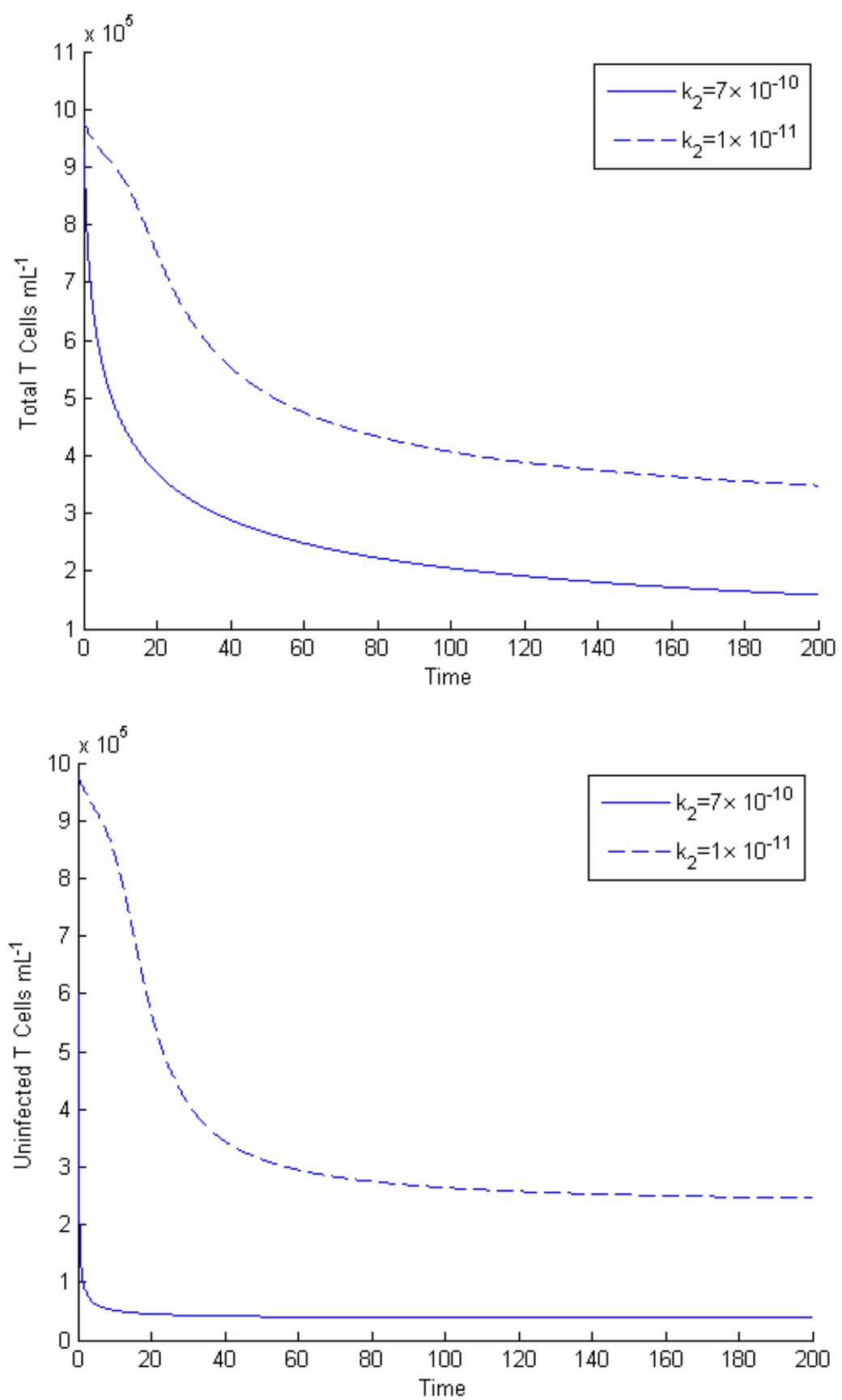

Figure 5: Dynamics of the total number and uninfected $T$ cells of system (3) for different values of $k_{2}$, the probability of cell-to-cell transmission, for $\alpha=0.5$, given parameter values in Table 2, and given initial conditions. For more information, see text. 

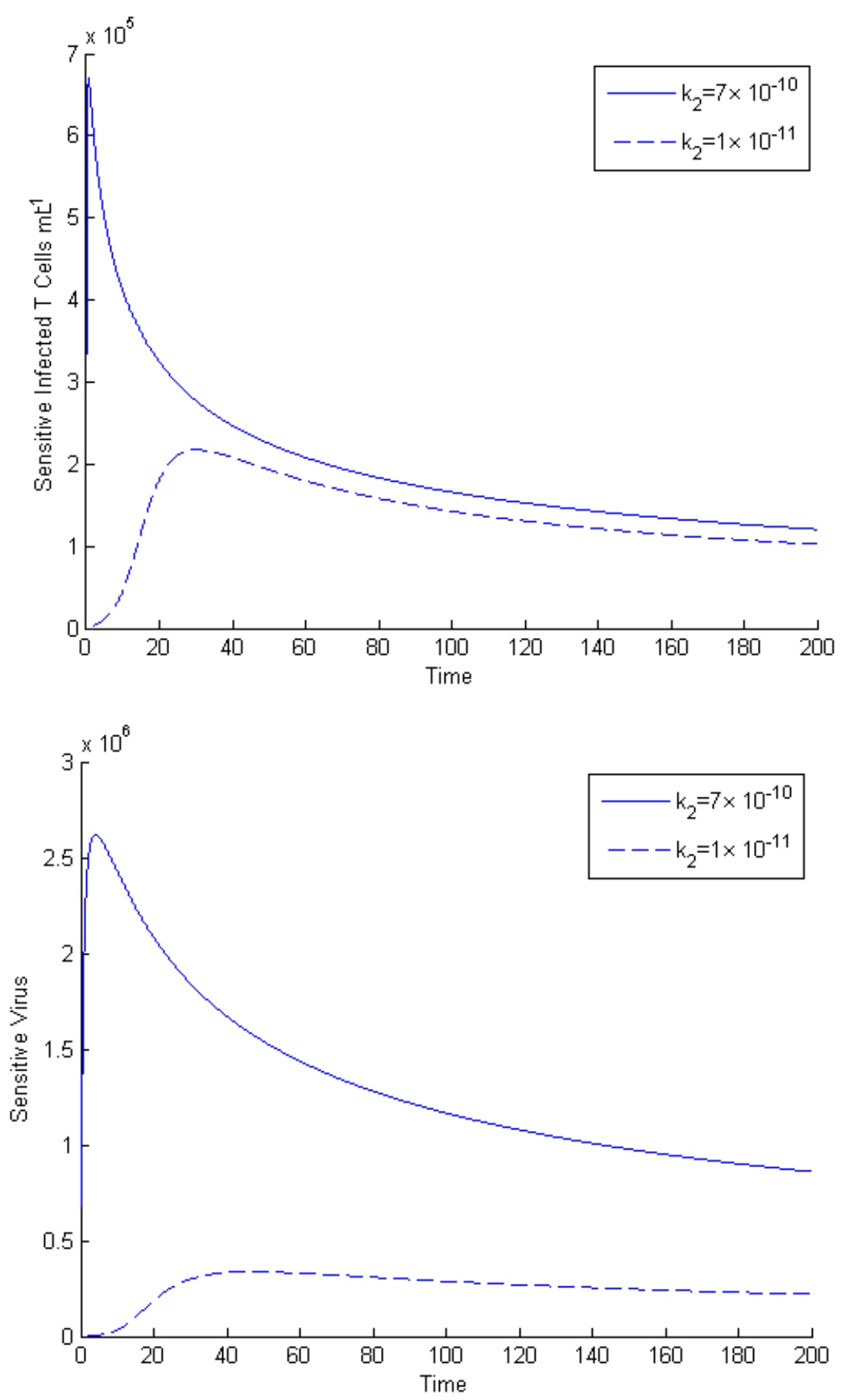

Figure 6: Dynamics of the sensitive $T$ cells and virus of system (3) for different values of $k_{2}$, the probability of cell-to-cell transmission, for $\alpha=0.5$, given parameter values in Table 2, and given initial conditions. For more information, see text. 


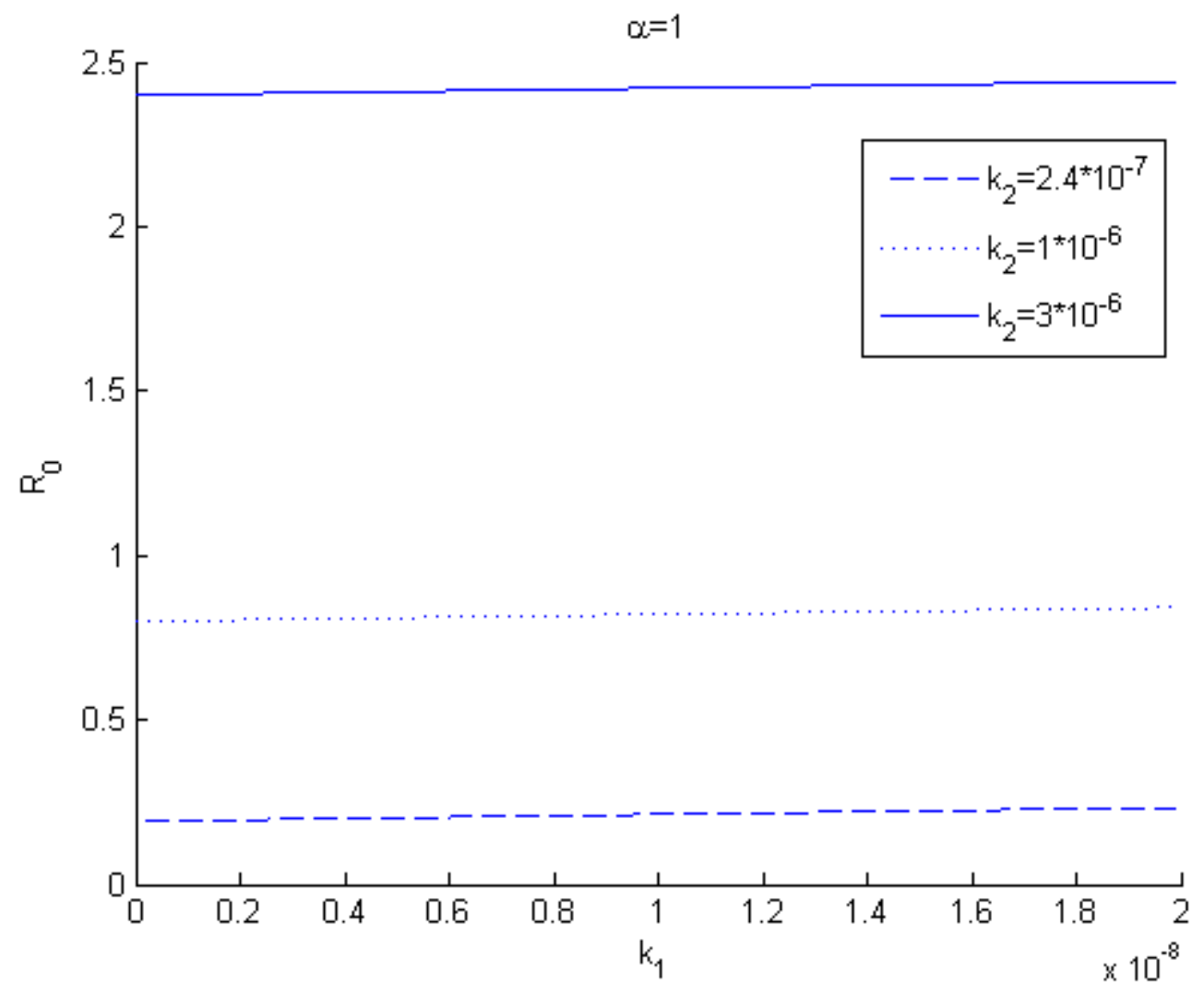

Figure 7: Variation of the value of the reproduction number $R_{0}$ as a function of the probabilities of cell-to-cell and virus-to-cell transmission modes, $k_{2}$ and $k_{1}$, respectively, for given parameter values in Table 2 and given initial conditions, $\alpha=1$. 


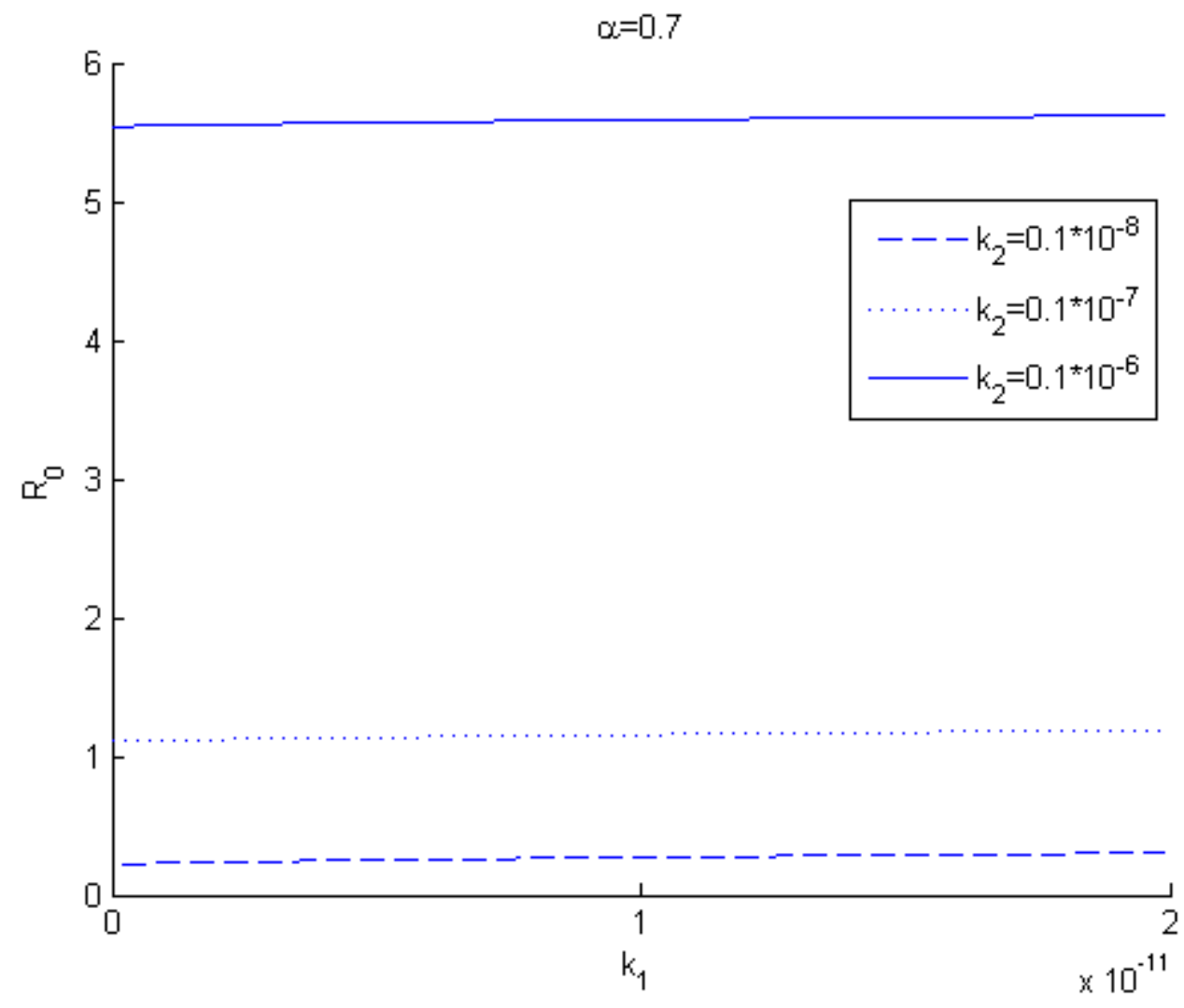

Figure 8: Variation of the value of the reproduction number $R_{0}$ as a function of the probabilities of cell-to-cell and virus-to-cell transmission modes, $k_{2}$ and $k_{1}$, respectively, for given parameter values in Table 2 and given initial conditions, $\alpha=0.7$. 


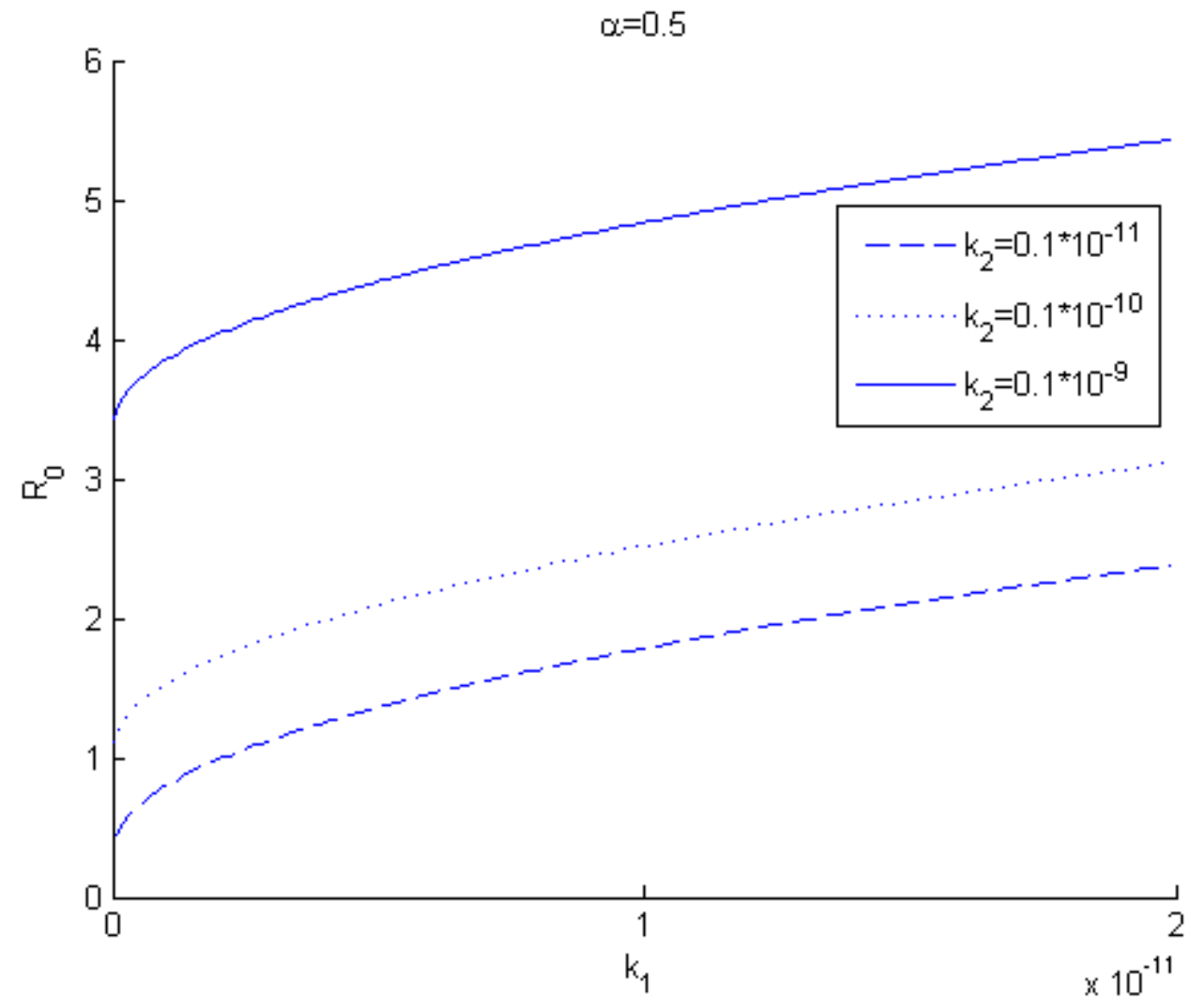

Figure 9: Variation of the value of the reproduction number $R_{0}$ as a function of the probabilities of cell-to-cell and virus-to-cell transmission modes, $k_{2}$ and $k_{1}$, respectively, for given parameter values in Table 2 and given initial conditions, $\alpha=0.5$. 

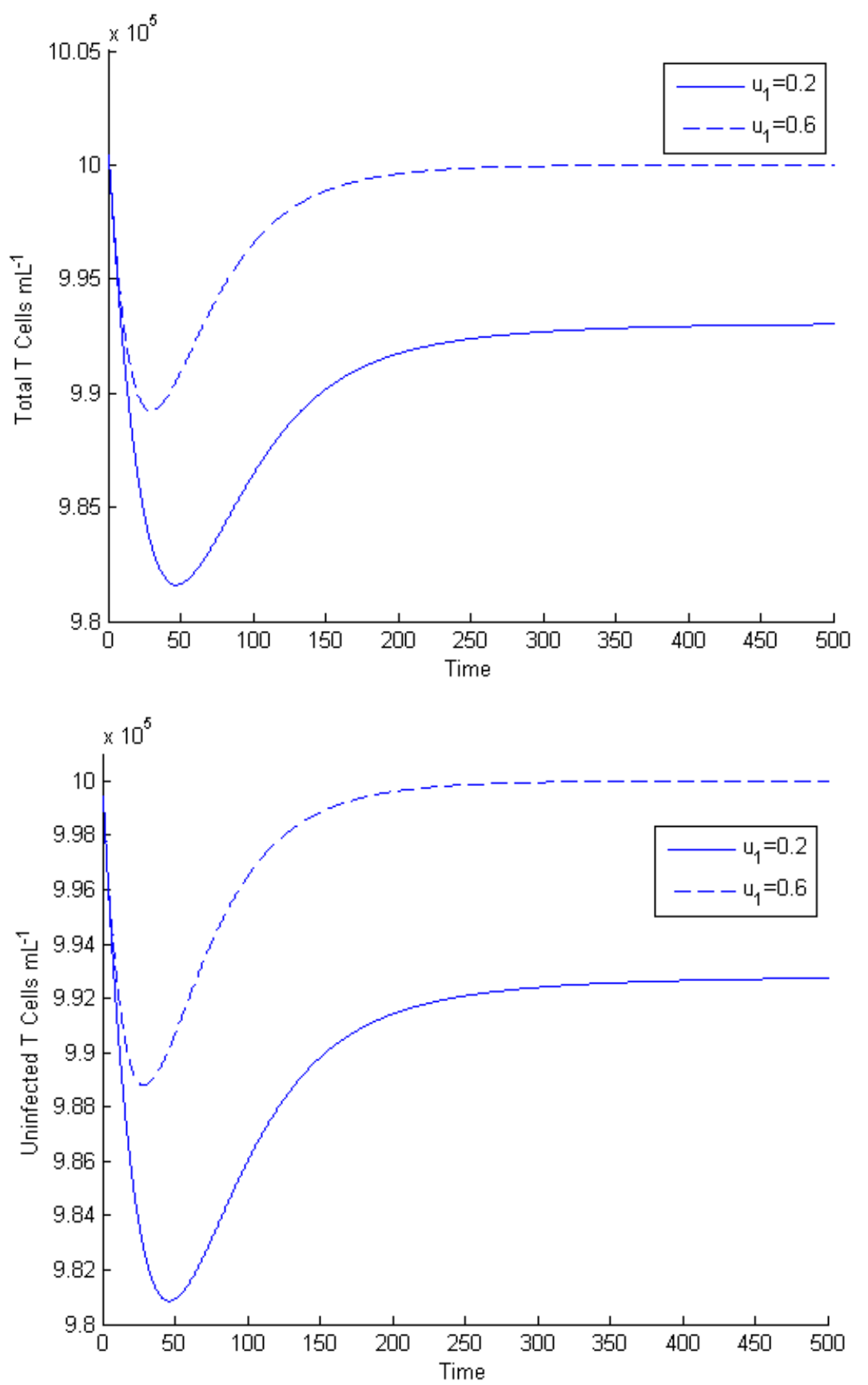

Figure 10: Dynamics of the total number and uninfected $T$ cells of system (3) for different values of $u_{1}$, the drug efficacy, for $\alpha=1$, given parameter values in Table 2 , except $k_{1}=1.5 \times 10^{-8}$ and $k_{2}=1.22 \times 10^{-6}$, and given initial conditions. 

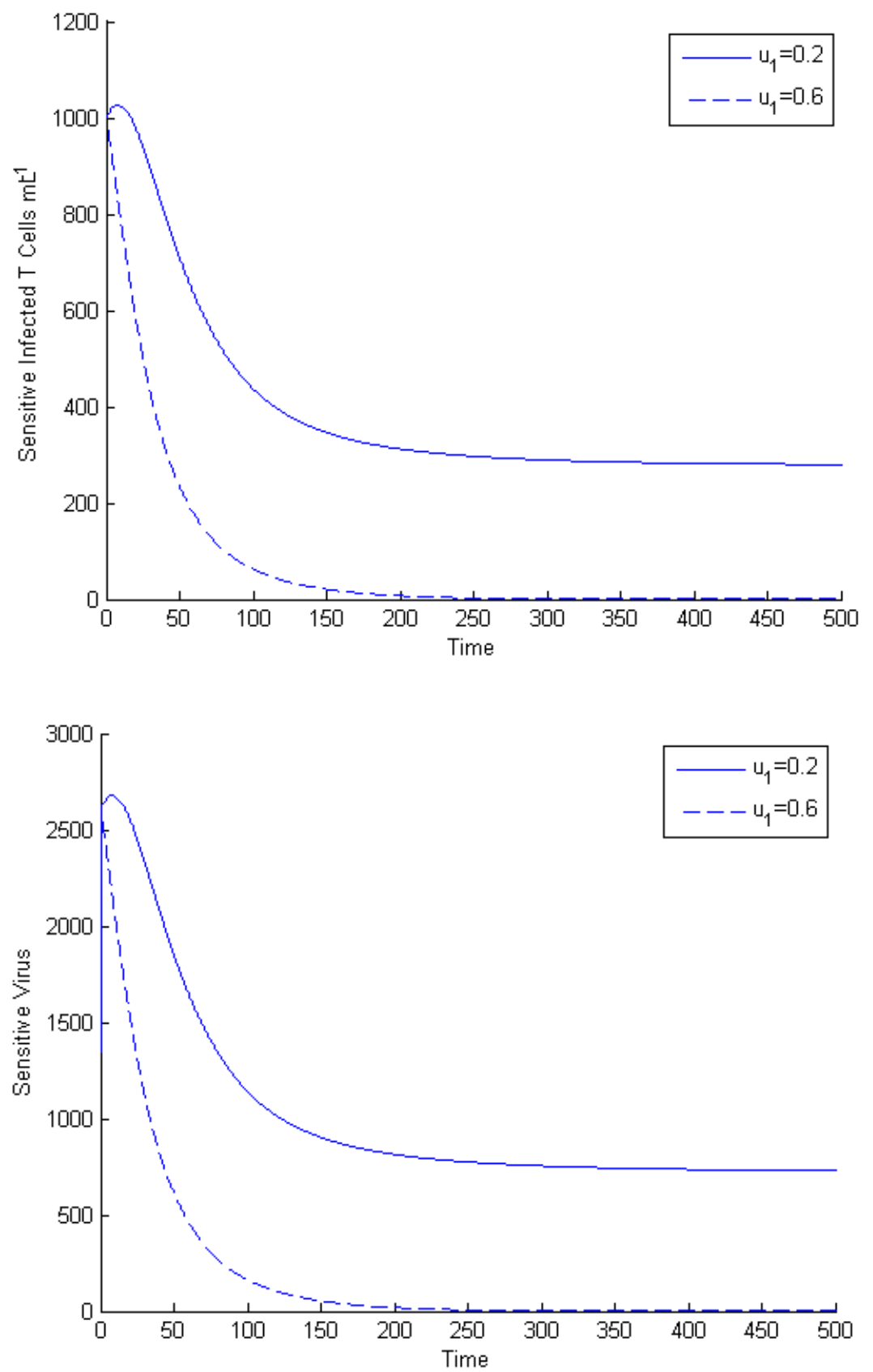

Figure 11: Dynamics of the sensitive $T$ cells and virus of system (3) for different values of $u_{1}$, the drug efficacy, for $\alpha=1$, given parameter values in Table 2 , except $k_{1}=1.5 \times 10^{-8}$ and $k_{2}=1.22 \times 10^{-6}$, and given initial conditions. 